\title{
A SURVEY OF THE THEORY OF SPECTRAL OPERATORS
}

\author{
NELSON DUNFORD \\ Dedicated to Marston Morse
}

1. Introduction and statement of the problem. The importance of the spectral reduction theory for bounded and unbounded selfadjoint and normal operators in Hilbert space is amply demonstrated by its diverse applications to such far reaching fields of mathematics as the theories of topological groups, almost periodic functions, harmonic analysis, and selfadjoint boundary value problems. The problems centering around the reduction theory for nonnormal operators are among the most important problems in the theory of linear operators. Notable among the many early contributions to such problems were those of I. Fredholm [20] in 1903 and G. D. Birkhoff [5] in 1908. Fredholm discussed a certain class of linear integral equations and Birkhoff, a class of linear differential boundary value problems on a finite interval. The operators discussed in the Fredholm theory are compact and have spectra which are at worst convergent sequences. The corresponding spectral resolutions need not be countably additive, or, what amounts to the same thing, the eigenvalue expansions need not be unconditionally convergent. The Fredholm theory was later given a more abstract basis, stated in operator form and free of determinant theory, by F. Riesz [32], J. Schauder [33 ], and T. H. Hildebrandt [22]. The deep and comprehensive work of Birkhoff on eigenvalue expansions associated with (not necessarily selfadjoint) differential operators of arbitrary order strongly suggests that, except for certain irregular cases, linear differential boundary value problems on a finite interval will have unconditionally convergent (in Hilbert space) eigenvalue expansions. That this is indeed the case is shown by the work of J. T. Schwartz ${ }^{1}$ and H. P. Kramer [24] who have given Birkhoff's results in an abstract linear operator form. The general formulation shows that the expansion theory is valid for operators whose analytical expressions may involve integral and difference operators as well as other types of terms. The recently announced results of M. A. Neumark $[28 ; 29 ; 30]$ on singular differ-

An address delivered before the Annual Meeting of the Society in Cincinnati on January 28, 1958 by invitation of the Committee to Select Hour Speakers for Western Sectional Meetings; received by the editors January 28, 1958.

${ }_{1}^{1}$ This, and other work of Schwartz referred to here will appear in Linear operations, Part II, by N. Dunford and J. T. Schwartz, a forthcoming volume to be published by Interscience Publishers. This volume will be referred to as L.O. II. See also [34]. 
ential operators of the second order suggests that a countably additive spectral reduction should be expected for a large class of $n$th order linear differential boundary value problems on the infinite interval. Schwartz has developed and applied operator theory to the extent of being able to give an operator-theoretic basis for many singular nonselfadjoint boundary value problems of the second order and in particular he has established a countably additive spectral reduction for the type of operators considered by Neumark. The analytical difficulties arising in the $n$th order case have retarded corresponding progress for the general problem. However, the research, as yet unpublished, of David McGarvey indicates that a large class of $n$th order differential operators with periodic coefficients will have a countably additive spectral reduction. It thus appears that enough evidence exists to justify a serious study, in abstract form, of operators which admit a countably additive spectral resolution. In fact, it has been conjectured by Schwartz that all singular linear differential boundary value problems on an infinite interval $I$ with very mild growth restrictions on the coefficients (except for those of a highly irregular or pathological character similar to the irregular cases of G. D. Birkhoff) determine, in a sense that will be made more precise presently, operators in $L_{2}(I)$ which have a countably additive spectral resolution.

The main problem, of course, is that of discovering conditions on an operator which, on the one hand, are sufficient to insure the existence of a countably additive resolution of the identity and, on the other hand, are stated in a form that may be applied to the more concrete problems of mathematical study. A beginning has been made on this problem and on many related problems all pertaining to the study of operators with countably additive spectral resolutions defined on the Borel sets in the complex plane. In this lecture I shall try to survey the present state of knowledge concerning such operators, which, for brevity, I shall call spectral operators, and in particular I shall describe briefly some of the applications of the theory of spectral operators to boundary value problems.

A word about the spectral reduction problem as opposed to the general reduction problem may be in order. The general problem is that of finding all projections $E$ which reduce an operator $T$ i.e., which commute with it. For if $E$ and thus $E^{\prime}=I-E$ commutes with $T$ then the whole $B$-space $\mathfrak{X}$ in which $T$ operates is the direct sum of the invariant subspaces $E \mathbb{X}$ and $E^{\prime} \mathfrak{X}$ and the study of $T$ is reduced to the study of $T$ on the invariant subspaces. Such a formulation of the reduction problem is not one that will suit our purposes in the study 
of a spectral reduction of an operator. For example, the general problem when applied to the identity operator is that of finding all projections. Whereas, from the spectral point of view, the identity, whose spectrum is a single point, may not be reduced further. A precise formulation of the spectral reduction problem for a bounded linear operator is as follows. For each set $\delta$ in the family $B$ of Borel sets in the complex plane we wish to find a projection $E(\delta)$ which reduces a given operator $T$ in the complex Banach space $\mathfrak{X}$ and is such that the spectrum of the restriction $T \mid E(\delta) \mathfrak{X}$ of $T$ to $E(\delta) \mathfrak{X}$ is contained in the closure $\bar{\delta}$ of $\delta$, i.e.,

$$
T E(\delta)=E(\delta) T, \quad \sigma(T \mid E(\delta) \mathfrak{X}) \subseteq \bar{\delta}, \delta \in B .
$$

The map $\delta \rightarrow E(\delta)$ of the Boolean algebra $B$ into the Boolean algebra of projections $E(\delta)$ should be a homomorphism mapping the units in $B$ into 0 and $I$, i.e.,

$$
\begin{aligned}
& E(\phi)=0, \quad E(p)=I, \quad E\left(\delta^{\prime}\right)=E(\delta)^{\prime}, \\
& E(\delta \cap \sigma)=E(\delta) \wedge E(\sigma), \quad E(\delta \cup \sigma)=E(\delta) \vee E(\sigma),
\end{aligned}
$$

where we have written $\phi, p$ for the void set and the whole complex plane respectively, $\delta^{\prime}$ for the complement of $\delta$ in $p, A^{\prime}$ for the complementary projection $I-A$, and $A \vee B, A \wedge B$ for $A+B-A B, A B$ respectively. A third condition demanded of the projections $E(\delta)$ is that they be bounded in $\delta$, i.e.,

$$
|E(\delta)| \leqq M
$$

for some constant $M$ independent of the Borel set $\delta$. Finally it is required that $E(\delta)$ be countably additive in $\delta$ in the strong operator topology, i.e., for every sequence $\left\{\delta_{n}\right\}$ of disjoint Borel sets

$$
E\left(\bigcup_{n=1}^{\infty} \delta_{n}\right) x=\sum_{n=1}^{\infty} E\left(\delta_{n}\right) x, \quad x \in \mathfrak{X} .
$$

(According to a theorem of Orlicz-Pettis [31] this condition of countable additivity will hold if each of the scalar functions $x^{*} E(\delta) x$ with $x$ in $\mathfrak{X}$ and $x^{*}$ in $\mathfrak{X}^{*}$ is countably additive on $B$.) The conditions (i), . . (iv) are clearly redundant but that does not concern us here. They state in clear form the four basic properties of the map $E: \delta \rightarrow E(\delta)$ which, since it is uniquely determined by $T$, is called the resolution of the identity for $T$ or the spectral resolution of $T$. The spectral reduction problem for an operator $T$ in a complex $B$-space $X$ is thus that of finding a resolution of the identity for $T$, i.e., a map $\delta \rightarrow E(\delta)$ of the Borel sets $\delta$ in the complex plane into a family of 
projections $E(\delta)$ having the properties (i), $\cdots$, (iv). An operator $T$ which has a spectral resolution in this sense is called a spectral operator. It has been observed [11] that the spectral reduction problem is, for finite dimensional spaces equivalent to the canonical reduction of Jordan in classical matrix theory. Thus every operator in a finite dimensional space is a spectral operator. The spectral theorem in Hilbert space shows that every bounded selfadjoint or normal operator is a spectral operator. In the preceding formulation of the spectral reduction problem the operator $T$ was tacitly assumed to be bounded. In case $T$ is merely a closed linear operator with domain $\mathfrak{D}(T)$ in $\mathfrak{X}$ the condition (i) must be replaced by the condition (i)' that follows.

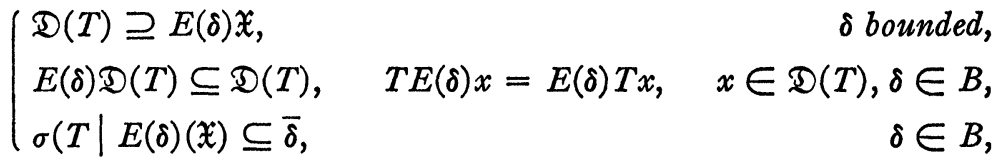

where the restriction $T \mid E(\delta) \mathfrak{X}$ of $T$ to $E(\delta) \mathfrak{X}$ has domain $\mathfrak{D}(T)$ $\cap E(\delta) \mathfrak{X}$. As in the bounded case the spectral resolution is uniquely determined ${ }^{2}$ by $T$. Well known examples of unbounded spectral operators are the unbounded selfadjoint operators in Hilbert space.

Besides the finite matrices and the selfadjoint or normal operators in Hilbert space I shall list here a few other examples of bounded and unbounded spectral operators of frequent occurrence in mathematical analysis. K. O. Friedrichs [21] has shown that the operator

$$
(T f)(s)=s f(s)+\int_{a}^{b} K(s, t) f(t) d t,
$$

where the kernel $K$ satisfies certain Lipschitz conditions, is actually similar to the selfadjoint operation $(A f)(s)=s f(s)$ in $L_{2}(a, b)$ and thus $T$ has a spectral resolution. The same result probably holds if $T$ is regarded as an operator in $L_{p}(a, b)$ with $1<p<\infty$ but, as far as I know, the details have not been checked. Friedrich suggests in his work that his result is also valid if, instead of assuming the Lipschitz conditions on $K$, it is assumed that $K$ belongs to a certain class of Fourier transforms. This suggestion was carried out by J. T. Schwartz who found that the operator $T$ of equation (v) is a spectral operator in $L_{p}(a, b)$ with $1<p<\infty$ provided that the kernel $K$ is the Fourier transform of a Borel measure $\mu$ in the plane whose total variation is less than $(2 \pi)^{-1}$. That is, the operator $T$ of equation (v) is a spectral operator in $L_{p}(a, b)$ with $1<p<\infty$ provided that

2 Bade [1] has developed the theory of unbounded spectral operators. Further results will be found in [15]. 


$$
K(s, t)=\int_{-\infty}^{\infty} \int_{-\infty}^{\infty} e^{i(s u+t v)} \mu(d u, d v), \quad \operatorname{var}(\mu)<1 / 2 \pi .
$$

An analogous result is true on the infinite interval. More precisely, if multiplication $(A f)(s)=s f(s)$ is defined on the domain $\mathfrak{D}(A)$ of those $f$ in $L_{p}(-\infty, \infty)$ for which

$$
\int_{-\infty}^{\infty}|s f(s)|^{p} d s<\infty
$$

and if $K$ is a kernel satisfying (vi) and the further restriction that the operations

$$
(K f)(s)=\int_{-\infty}^{\infty} K(s, t) f(t) d t, \quad(L f)(t)=\int_{-\infty}^{\infty} K(s, t) f(s) d s
$$

are bounded linear operations in $L_{p}(-\infty, \infty)$ then the operation

$$
(T f)(s)=s f(s)+\int_{-\infty}^{\infty} K(s, t) f(t) d t
$$

is, for $1<p<\infty$, an unbounded spectral operator in $L_{p}(-\infty, \infty)$ with domain $\mathfrak{D}(A)$ which is similar to the operator $A$.

The results stated in the preceding paragraph may be proved by Friedrich's methods of establishing similarity of operators. Another method which has been useful in establishing the existence of a spectral resolution for nonselfadjoint operators consists of determining analytical conditions on the resolvent of an operator which are sufficient to insure the existence of projections $E(\delta)$ satisfying conditions (i), $\cdots$, (iv). This method, which will be indicated in some detail in $\S \S 3$ and 4 that follow, leads to the surprising result that, for large classes of operators which occur among the natural objects of mathematical study, the condition on the resolvent which states the boundedness condition (iii) is by itself sufficient to insure that the operator is a spectral operator. It should be mentioned that the analytical forms taken on by the boundedness condition (iii) in the various applications given explicitly later, are by no means easily applied in all examples. In fact only in the known case of selfadjoint operators in Hilbert space is it clearly satisfied. In all other cases where the condition has been verified the analytical calculations have been considerable. A few examples of differential operators for which Schwartz has verified the boundedness condition are the following. Other examples have been recently discovered by D. R. Smart. ${ }^{3}$

${ }^{3}$ Communicated to the author by letter. 
In the case of differential operators associated with boundary value problems on a finite interval the situation is the following. The unbounded operator $T$ in $L_{2}(a, b)$ determined by the formal differential expression

$$
(T f)(t)=f^{(n)}(t)+\sum_{j=0}^{n-1} a_{j}(t) f^{(j)}(t),
$$

where the $a_{j}$ 's are arbitrary bounded, complex, measurable functions, if restricted by a set of linearly independent, homogeneous, and regular (in the sense of Birkhoff) boundary conditions, is a spectral operator in $L_{2}(a, b)$. In this case the resolvent is compact and thus the spectrum is discrete and only a finite number of the eigenvalues have index ${ }^{4}$ greater than one. The associated generalized ${ }^{5}$ eigenvalue expansion is unconditionally convergent. All of these properties hold for a somewhat more general class of unbounded operators, e.g., operators $T$ in $L_{2}(a, b)$ having the form

$$
(T f)(t)=f^{(n)}(t)+\sum_{j=0}^{n-2} B_{j} f^{(j)}
$$

where the $B_{j}$ 's are arbitrary bounded linear operators in $L_{2}(a, b)$. The operators $B_{j}$ may be integral or difference operators or a combination of such and thus there is a large class of linear $n$th order boundary value problems in a finite interval which determine spectral operators.

As mentioned earlier Neumark has recently announced results which, in our terminology, amount to the assertion that certain singular second order differential operators are spectral operators. Following an idea suggested by Neumark, Schwartz has verified the conditions of the general theory of spectral operators and thus has put the Neumark type of singular differential operator into the framework of general spectral theory. Briefly (and somewhat incompletely) stated the Neumark-Schwartz result (or rather one of the numerous such results) is that the unbounded operator $T$ in $L_{2}(0, \infty)$ determined by the formal differential operator

$$
\tau=-(d / d t)^{2}+q(t),
$$$$
0 \leqq t<\infty
$$

and an arbitrary nontrivial linear homogeneous boundary condition at zero, where $q$ is a bounded complex valued measurable function for which

4 The index of an eigenvalue $\lambda$ is the smallest non-negative integer $n$ for which the equation $(T-\lambda I)^{n} x=0$ has the same solutions as the equation $(T-\lambda I)^{n+1} x=0$.

- A generalized eigenvalue expansion is one of the form $x=\sum x_{m}$ where $\left(T-\lambda_{m} I\right)^{n_{m}} x_{m}=0$. 


$$
\int_{0}^{\infty}\left(1+t^{2}\right)|q(t)| d t<\infty
$$

is a spectral operator. Basically this is a result on the perturbation of spectral operators with continuous spectrum. Closely related results for the selfadjoint case were established by Moser [27]. The main part of the analytical work involved in verifying the conditions required by the general theory is in obtaining sufficiently good asymptotic estimates for the solutions of the equation $\tau f=\lambda f$.

In formulating the spectral reduction problem as we have done by demanding boundedness and countable additivity of the spectral resolution we have admittedly ruled out many interesting and elementary operators. For example Wermer [38] has shown that the map $\left\{\alpha_{n}\right\} \rightarrow\left\{\alpha_{n+1}\right\}$ in $l_{2}(0, \infty)$ is reduced by no bounded projection other than 0 and $I$. Fixman ${ }^{6}$ has recently shown that even the unitary shift operator in $l_{p}(-\infty, \infty)$ fails, in case $p \neq 2$, to have a countably additive resolution of the identity. Fixman has also given examples of unitary operators in $C(\Omega)$, the complex continuous functions on the compact Hausdorff space $\Omega$, which are not spectral operators. In fact by demanding a countably additive resolution of the identity we probably rule out most of the differential operators in $L_{p}$ with $p \neq 2$. However in many of the irregular cases of eigenvalue expansions associated with boundary value problems with discrete spectrum where the expansions are not unconditionally convergent (and thus the operators are not spectral operators) the general theory of spectral operators shows exactly how the expansion may be summed to the function being expanded.

A word about the organization of the present communication. In $\$ 2$ the properties of spectral operators, their multiplicity theory, and the theory of algebras of spectral operators will be very briefly described. Practically no proofs will be given here as they are either to be found in the literature ${ }^{7}$ or else they will appear in papers by Bade [4] or Foguel $[17 ; 18 ; 19]$. In $\$ \S 3$ and 4 however, we present in some detail, giving proofs and complete statements, the converse problem i.e., the problem of determining conditions on an operator sufficient to make it a spectral operator. In $\$ 3$ four properties of the analytic functions $(\xi I-T)^{-1} x$ and their singularities are stated which are necessary and sufficient in order that an operator in a weakly complete space be a spectral operator. Because of their generality these conditions are quite difficult to apply in most concrete cases and for

\footnotetext{
${ }^{6}$ Communicated to the author by letter.

7 Most of the results mentioned in $\$ 2$ will be found in the references $[1 ; 2 ; 3 ; 13$; $16 ; 23 ; 37]$.
} 
this reason we discuss, in $\S 4$, operators whose spectra lie in Jordan curves and whose resolvents have a finite rate of growth along a set of transversals to the Jordan curves. In this situation all but one of the four conditions required in $\$ 3$ may be proved in abstract form and thus there is but one (the boundedness condition (B) of $\$ 3$ which corresponds to (iii) above) to be verified in specific cases. The restrictions on the topological nature of the spectrum and on the rate of growth of the resolvent which are imposed in $\$ 4$ are justified by the fact that they are satisfied by most operators arising from differential boundary value problems.

In fact the $n$th order singular differential operators with periodic coefficients have been shown by McGarvey to have their spectra lying in a finite set of analytic arcs. Also G. C. Rota has recently shown that the $n$th order singular operators whose coefficients are rational functions have their essential spectra in a finite set of analytic arcs. The resolvent in these cases has first order rate of growth along the normals to interior points of these analytic arcs. In these situations one finds examples of spectral operators which, unlike the situations considered by Neumark, are not similar to selfadjoint operators. For example McGarvey has shown that the second order operator $-(d / d t)^{2}+a(d / d t)+q(t)$ where $a$ is a real constant not zero and $q$ is a complex valued periodic function small relative to $a$ determines a spectral operator in $L_{2}(-\infty, \infty)$. In this situation the spectrum need not be real and the operator need not be similar to a selfadjoint operator. If $a=0$ this is no longer true for McGarvey shows that the operator $-(d / d t)^{2}+\epsilon e^{2 \pi i t}$ is not spectral for any $\epsilon \neq 0$. What destroys the spectral property in those cases which have been analyzed is the existence of a finite set of irregular branch points about which the spectral resolution is not countably additive.

A number of examples such as those studied by McGarvey, Neumark, and Rota, has led Schwartz to conjecture that the spectrum of a singular differential operator under quite general restrictions on the rate of growth of its coefficients and quite general boundary conditions will consist of a finite or enumerable number of analytic arcs running into and out of a finite or enumerable set of branch points, together with an enumerable, or vacuous, set of point eigenvalues whose only limit points are the branch points. It is further conjectured that the resolvent will have first order rate or growth along the normals to points interior to the analytic arcs and that the corresponding operators will be spectral operators in the complement of that portion of $L_{2}$ which is associated with an arbitrarily small neighborhood of the branch points. 
Thus the theory in $\S \S 3$ and 4 is presented in all detail in the hopes that it will interest others in the problem of verifying the Schwartz conjecture.

2. Properties of spectral operators. Before seeking conditions on the resolvent $R(\xi ; T)$ of a linear operator $T$ in a complex $B$-space $\mathfrak{X}$ which are sufficient to insure that $T$ is a spectral operator we shall discuss here some of the properties of spectral operators and in particular see that the resolvent of such an operator does have properties not enjoyed by all resolvents. In other words, before seeking conditions sufficient for a spectral reduction we shall seek necessary conditions. Many of the results discussed are to be found in the literature or else will appear shortly in papers by S. R. Foguel and so proofs will be omitted. However a proof will be given for the canonical reduction of a spectral operator as it is more transparent than the one in the literature. ${ }^{8}$ Recently a similar proof was communicated to me by Ciprian Foias.

Throughout this section $T$ will be assumed to be a spectral operator in the complex $B$-space $\mathfrak{X}$. There are three properties of the resolvent $R(\xi ; T)=(\xi I-T)^{-1}$ of the spectral operator $T$ which are not properties of all resolvent operators but which are the basic properties of spectral operators that I wish to emphasize. In the following section it will be seen that these three properties come near to being sufficient for a spectral reduction and that for the operations arising in many mathematical problems the conditions (A) and (C) are automatically satisfied and only the boundedness condition (B) remains to be verified. The first of these properties is the single valued extension property.

(A) For each $x$ in $X$ the function $R(\xi ; T) x$ has the single valued extension property.

To interpret this statement a vector valued function $f$ is called an extension of $R(\xi ; T) x$ if it is defined and analytic on an open set $D(f)$ containing the resolvent set $\rho(T)$ of $T$ and if for each $\xi$ in $D(f)$ we have $(\xi I-T) f(\xi)=x$. The function $R(\xi ; T) x$ is said to have the single valued extension property if every pair $f, g$ of extensions of $R(\xi ; T) x$ have $f(\xi)=g(\xi)$ for $\xi$ in $D(f) \cap D(g)$. The union of all the open set $D(f)$ as $f$ varies over all extensions of $R(\xi ; T) x$ is called the resolvent set of $x$ and is denoted by the symbol $\rho(x)$. The spectrum $\sigma(x)$ of $x$ is defined as the complement in the complex plane of the resolvent set of $x$. In view of (A) it is clear that there is a uniquely defined maximal extension $x(\xi), \xi \in \rho(x)$ of $R(\xi ; T) x$.

\footnotetext{
8 Theorem 8 in [13].
} 
The second basic property of spectral operators is the boundedness condition expressed in the statement (B) that follows.

(B) There is a constant $K$ depending only upon $T$, such that for every pair $x, y$ of vectors with $\sigma(x), \sigma(y)$ disjoint we have

$$
|x| \leqq K|x+y| \text {. }
$$

The third and final condition that is to be stressed is the following closure property.

(C) For every closed set $\delta$ of complex numbers the set of all vectors $x$ with $\sigma(x) \subseteq \delta$ is also closed.

The set of vectors $x$ whose spectrum lies in a given closed set $\delta$ is not only closed but it is precisely the range of the projection $E(\delta)$ associated with $\delta$ by the spectral resolution of $T$. It follows as a corollary that every bounded operator which commutes with $T$ also commutes with every spectral resolution for $T$ and this shows that the spectral resolution is uniquely determined by $T$. It should also be mentioned that $E(\sigma(T))=I$ and that $\sigma(x)$ is void if and only if $x=0$.

There is a canonical reduction for spectral operators which corresponds to the Jordan reduction for finite matrices of complex numbers. To explain this reduction we introduce the concept of a scalar type operator as an operator $S$ which has a spectral resolution $E$ for which

$$
S=\int \lambda E(d \lambda) .
$$

This canonical reduction asserts that an operator $T$ is a spectral operator if and only if it is the sum $T=S+N$ of a scalar type operator $S$ and a quasi-nilpotent operator $N$ commuting with $S$. Furthermore this decomposition is unique and $T$ and $S$ have the same spectrum and the same spectral resolution. The operator $S$ is called the scalar part of $T$ and $N$ the radical part of $T$.

Here we have used the term quasi-nilpotent for an operator $N$ for which $\left|N^{n}\right|^{1 / n} \rightarrow 0$. It is clear that this condition is equivalent to the statement that the Laurent expansion for the resolvent $\Sigma N^{n} / \lambda^{n+1}$ $=R(\lambda ; N)$ is convergent for every $\lambda \neq 0$. Thus the operator $N$ is quasi-nilpotent if and only if its spectrum $\sigma(N)=\{0\}$. S. R. Foguel [17] has shown that the scalar part $S$ of a spectral operator $T$ inherits many of the properties of $T$ but it need not inherit the fine structure properties of the spectrum. One of Foguel's principal results asserts that if $\Re$ is a uniformly closed right (or left) ideal in the algebra of bounded operators on $\mathfrak{X}$, and if $T$ belongs to $\Re$ then so do $S, N$, 
and every projection $E(\delta)$ for which $0 \notin \bar{\delta}$. A number of interesting corollaries are immediate, e.g.: if $T$ is compact so are $S, N$, and $E(\delta), 0 \notin \bar{\delta}$ compact; if $T$ is weakly compact so are $S, N$, and $E(\delta)$, $0 \notin \bar{\delta}$ weakly compact; the ranges of $S, N$, and $E(\delta), 0 \notin \bar{\delta}$ are contained in the closure of the range of $T$-thus if $T$ is separable so are $S, N$, and $E(\delta), 0 \notin \bar{\delta}$ separable; a similar result, also proved by Foguel, although not an immediate corollary of the above principle, asserts that $S$ has a closed range if $T$ does. Among other corollaries we mention the following: if $A T=0$ then $A S=A N=A E(\delta)=0,0 \notin \bar{\delta}$; if $T x=0$ then $N x=S x=E(\delta) x=0,0 \notin \bar{\delta}$; if for some bounded sequence $\left\{x_{n}\right\}$ the sequence $\left\{T x_{n}\right\}$ is convergent then if $0 \notin \bar{\delta}$ the sequences $\left\{S x_{n}\right\},\left\{N x_{n}\right\},\left\{E(\delta) x_{n}\right\}$ are also convergent.

Foguel has also shown that for a bounded operator $A$, we have $T A=0$ if and only if $A=E(\{0\}) A$ and $N E(\{0\}) A=0$. It follows that if $E(\{0\})=0$ then $T A=0$ or $A T=0$ only when $A=0$ and that $\overline{T \mathfrak{X}}=\mathfrak{X}$. The following elementary examples are used by Foguel to show that the converses to a number of the above mentioned results are false. The operator $T=S+N$ in $l_{1}$ is defined by placing $S=0$ and $N\left(\xi_{1}, \xi_{2}, \cdots\right)=\left(\xi_{2}, 0, \xi_{4}, 0, \cdots\right)$. Then $S$ is compact but $T$ is not even weakly compact. In the space $C_{0}(0,1)$, of continuous functions vanishing at the origin, let $T=S+N$ with $S=0$ and $(N f)(t)=\int_{0}^{t} f(s) d s$. Then $S$ has a closed range but $T$ does not and here the point spectrum of $S$ is the continuous spectrum of $T$. If, in this last example, we take $S$ to be $I$ instead of 0 then the ranges of $T$ and $S$ are both closed but the range of $N$ is not closed.

Returning for a moment to the canonical reduction itself we shall indicate here an elementary proof which is independent of Gelfand's theory of normed rings. It will first be shown that the sum $T=S+N$ of a scalar operator $S$ and a quasi-nilpotent $N$ commuting with $S$ is a spectral operator having the same spectral resolution as $S$. Let $E$ be the spectral resolution for $S$ so that for each Borel set $\delta$ the projection $E(\delta)$ commutes with $N$ and thus with $T$. Thus to show that $E$ is also a spectral resolution for $T$ it suffices to show that $\sigma(T \mid E(\delta) \mathfrak{X})$ $\subseteq \bar{\delta}$ for every Borel set $\delta$. But, since $E$ is a spectral resolution for $S$ this will follow if it is shown that $\sigma(T \mid E(\delta) \mathfrak{X})=\sigma(S \mid E(\delta) \mathfrak{X})$. This latter fact follows (by replacing $\mathfrak{X}$ by $E(\delta) \mathfrak{X}$ ) from the equation $\sigma(S+N)=\sigma(S)$ which will now be derived in an elementary fashion rather than by appealing to the ideal theory in normed rings. Since $N$ is quasi-nilpotent it follows that $\left|N^{k}\right|=O\left(\epsilon^{k}\right)$ for every $\epsilon>0$ and thus for every $\lambda$ in $\rho(S)$ the series $\sum_{k=0}^{\infty} N^{k} R(\lambda ; S)^{k}$ converges in the uniform operator topology. Since 


$$
\begin{array}{r}
\left\{\sum_{k=0}^{\infty} N^{k} R(\lambda ; S)^{k}\right\}\{I-N R(\lambda ; S)\}=\{I-N R(\lambda ; S)\} \sum_{k=0}^{\infty} N^{k} R(\lambda ; S)^{k} \\
=\sum_{k=0}^{\infty} N^{k} R(\lambda ; S)^{k}-\sum_{k=1}^{\infty} N^{k} R(\lambda ; S)^{k}=I,
\end{array}
$$

the series is $\{I-N R(\lambda ; S)\}^{-1}$. This shows that

$$
(\lambda I-S-N)^{-1}=R(\lambda ; S)\{I-N R(\lambda ; S)\}^{-1}
$$

exists as an everywhere defined and bounded operator. Thus $\lambda \in \rho(S+N)$ which shows that $\sigma(S) \supseteq \sigma(S+N)$. It follows as a corollary that $\sigma(S+N) \supseteq \sigma(S+N-N)=\sigma(S)$ and so $\sigma(S+N)=\sigma(S)$. Thus $T$ is a spectral operator having the same spectral resolution as $S$. Since $S=\int \lambda E(d \lambda)$ it follows that $S$ is uniquely determined by $T$. Hence $N=T-S$ is also uniquely determined by $T$.

Finally it will be shown that every spectral operator $T$ has the desired decomposition. The operators $S$ and $N$ are defined by the equations

$$
S=\int \lambda E(d \lambda), \quad N=T-S
$$

where $E$ is the resolution of the identity for $T$. It is clear that $S$ is a scalar type operator with resolution of the identity $E$. Also, since $T$ commutes with $E(\delta)$ it commutes with $S$ and thus $N$ commutes with $S$. The desired conclusion will therefore be established as soon as it is shown that $N$ is a quasi-nilpotent. To prove this we will show that the spectrum $\sigma(N)$ of $N$ is contained in the circle $C_{\mathbf{e}}=\{\lambda|| \lambda \mid \leqq \epsilon\}$ whose radius $\epsilon$ is an arbitrary positive number. Now let the spectrum of $T$ be decomposed into the union of the disjoint Borel sets $\sigma_{1}, \cdots, \sigma_{k}$ each having diameter less than a positive number $\alpha<\epsilon$ which will be specified presently. If $\lambda$ is in the resolvent set of each of the restrictions $N_{\sigma_{i}}=N \mid E\left(\sigma_{i}\right)$ X, and if $R_{i}=R\left(\lambda, N_{\sigma_{i}}\right)$, then, putting $R=\sum_{i=1}^{k} R_{i} E\left(\sigma_{i}\right)$, we have

$$
(\lambda I-N) R=\sum_{i=1}^{k}\left(\lambda I-N_{\sigma_{i}}\right) R_{i} E\left(\sigma_{i}\right)=\sum_{i=1}^{k} E\left(\sigma_{i}\right)=I
$$

and

$$
\begin{aligned}
R(\lambda I-N) & =\sum_{i=1}^{k} R(\lambda I-N) E\left(\sigma_{i}\right)=\sum_{i=1}^{k} R\left(\lambda I-N_{\sigma_{i}}\right) E\left(\sigma_{i}\right) \\
& =\sum_{i=1}^{k} R_{i}\left(\lambda I-N_{\sigma_{i}}\right) E\left(\sigma_{i}\right)=\sum_{i=1}^{k} E\left(\sigma_{i}\right)=I
\end{aligned}
$$


Thus $\lambda$ is in $\rho(N)$. Consequently, the spectrum of $N$ is contained in the union of the spectra $\sigma\left(N_{\sigma_{i}}\right)$ of the restrictions $N \mid E\left(\sigma_{i}\right)$, so that it will suffice to show that $\sigma\left(N_{\sigma_{i}}\right) \subseteq C_{\epsilon}$ for each $i=1, \cdots, k$. To show this we write

$$
N_{\sigma_{i}}=\left(T-\lambda_{i} I\right)_{\sigma_{i}}+\left(\lambda_{i} I-S\right)_{\sigma_{i}}
$$

where $\lambda_{i}$ is a point in $\sigma\left(T_{\sigma_{i}}\right)$. Since $\sigma\left(T_{\sigma_{i}}\right) \subseteq \bar{\sigma}_{i}$ we have,

$$
\sigma\left(\left(T-\lambda_{i} I\right)_{\sigma_{i}}\right) \subseteq \bar{\sigma}_{i}-\lambda_{i} \subseteq C_{\alpha} \subseteq C_{\mathrm{e}} .
$$

Since $\left(\lambda_{i} I-S\right)_{\sigma_{i}}$ is the restriction of $\int_{\sigma_{i}}\left(\lambda_{i}-\lambda\right) E(d \lambda)$ to $\sigma_{i}$ we have

$$
\left|\left(\lambda_{i} I-S\right)_{\sigma_{i}}\right| \leqq v(E) \max _{\lambda \in \sigma_{i}}\left|\lambda-\lambda_{i}\right| \leqq \alpha v(E)
$$

and thus $\left(\lambda_{i} I-S\right)_{\sigma_{i}}$ is small in norm if $\alpha$ is small. Thus $\sigma\left(N_{\sigma_{i}}\right) \subseteq C_{\mathrm{e}}$ for small $\alpha$. By the above, this shows that $\sigma(N) \subseteq C_{\epsilon}$ and since $\epsilon>0$ is arbitrary it follows that $\sigma(N)=\{0\}$. It then follows that $N$ is a quasi-nilpotent. Q.E.D.

For functions $f$ in the algebra $\varsubsetneqq(T)$ of all complex functions defined, single valued, and analytic on an open set containing the spectrum $\sigma(T)$ of $T$ the formula

$$
f(T)=\frac{1}{2 \pi i} \int_{C} f(\lambda) R(\lambda ; T) d \lambda,
$$

where $C$ is a contour surrounding the spectrum of $T$, establishes a homomorphic map of $F(T)$ into the algebra of operators on $\mathfrak{X}$ which maps 1 into $I$ and $\lambda$ into $T$. Thus it establishes an operational calculus. For a spectral operator $T$ with radical part $N$ the formula (i) may be written as

$$
f(T)=\sum_{n=0}^{\infty} \frac{N^{n}}{n !} \int f^{(n)}(\lambda) E(d \lambda),
$$

the series being convergent in the uniform operator topology. Or, since $f^{(n)}(S)=\int f^{(n)}(\lambda) E(d \lambda)$, it may be written as

$$
f(S+N)=\sum_{n=0}^{\infty} \frac{N^{n}}{n !} f^{(n)}(S)
$$

a form which, as Schwartz has shown, [35] is applicable to any pair of commuting operators $S$ and $N$ provided that $f$ is single valued and analytic on an open set containing the spectra $\sigma(S)$ and $\sigma(S+N)$. If, for each $f$ in $F(T)$, the formula (ii) reduces to

$$
f(T)=\sum_{m=0}^{n} \frac{N^{m}}{m !} \int f^{(m)}(\lambda) E(d \lambda),
$$


the operator $T$ is said to be of type $n$. The operator is of type $n$ if and only if $N^{n+1}=0$ and it is a scalar operator if and only if it is of type zero. Foguel has shown that spectral operators of finite type can have no residual spectrum and that numbers $\lambda$ in the point spectrum are characterized by the condition $E(\{\lambda\}) \neq 0$. In general a spectral operator $T$ may have a residual spectrum and the relations

$$
\sigma_{c}(S) \subseteq \sigma_{c}(T), \quad \sigma_{p}(T) \cup \sigma_{r}(T) \subseteq \sigma_{p}(S)
$$

between the point, continuous, and residual spectra of $T$ and its scalar part $S$ have been established by Foguel.

J. Wermer [37] has shown that the scalar operators in Hilbert space are those operators which are similar to normal operators. More precisely, Wermer, using an idea of Mackey [26], which is based upon a powerful inequality of Lorch [25], has shown that for every finite set $S_{1}, \cdots, S_{k}$ of commuting scalar operators in Hilbert space there is a bounded selfadjoint operator $B$ with a bounded everywhere defined inverse such that the operators $B S_{i} B^{-1}$, $i$ $=1, \cdots, k$, are all normal. This shows that the sum and product of two commuting bounded spectral operators in Hilbert space are also spectral operators. As Kakutani [23] has shown this is not true in all $B$-spaces. In this connection however, it has been observed by Foguel [16] that in any space the sum (product) of two commuting spectral operators is a spectral operator if and only if the sum (product) of their scalar parts is a scalar type operator.

For applications to differential boundary value problems some of the properties of unbounded spectral operators must be developed. Most of the necessary work in this direction has been done by W. G. Bade [1]. We shall discuss here very briefly a few of the facts concerning unbounded operators. All proofs will be found either in Bade's work or in L.O. II.

While the theory of unbounded spectral operators is developed in the works cited $a b$ initio and not made to depend in a fundamental way on the theory of bounded spectral operators there is a fundamental connection between bounded and unbounded spectral operators which is helpful to keep in mind. If the complex number $\lambda$ is in the resolvent set of a closed operator $T$ then $T$ is a spectral operator if and only if its resolvent $R(\lambda ; T)$ is a spectral operator whose spectral resolution $E_{1}$ has $E_{1}(\{0\})=0$. Another useful property relating the concepts of bounded and unbounded spectral operators is the following. If $E$ is the spectral resolution for a closed spectral operator $T$ then the restriction of $T$ to $E(\delta) \mathfrak{X}$ is a spectral operator whose spectral resolution is the restriction of $E$ to $E(\delta) \mathfrak{X}$ and, in 
particular, if $\delta$ is bounded then the restriction of $T$ to $E(\delta) \mathfrak{X}$ is a bounded spectral operator. There is a commutativity property for unbounded closed spectral operators which is quite analogous in statement and proof to the one stated for bounded operators. Let $T$ be such an operator with spectral resolution $E$ and let $A$ be a bounded operator with $A \mathfrak{D}(T) \subseteq \mathfrak{D}(T)$ and $A T x=T A x$ for $x$ in $\mathfrak{D}(T)$. Then $A$ commutes with all the projections $E(\delta)$. Just as in the bounded case this property may be used to prove the uniqueness of the spectral resolution of a closed operator. Bade has given examples to show that the canonical reduction theorem is not valid for unbounded spectral operators. However, if $S$ is a closed scalar type spectral operator with spectral resolution $E$ and if $N$ is a bounded operator commuting with all the projections $E(\delta)$, then $S+N$ is a spectral operator with spectral resolution $E$ provided that the restriction of $N$ to each of the subspaces $E(\delta) \mathfrak{X}$, with $\delta$ bounded, is quasi-nilpotent.

An operational calculus exists for closed spectral operators and may be developed as follows. Let $E$ be the resolution of the identity for the closed spectral operator $T$ and let $f$ be single valued and analytic on an open set $G$ with $E(G)=I$. Let $\left\{\delta_{n}\right\}$ be an arbitrary increasing sequence of bounded Borel sets with closures contained in $G$ and such that $E\left(\bigcup_{n=1}^{\infty} \delta_{n}\right)=I$. Then the operator $f(T)$ is defined by the equations

$$
\begin{array}{rlrl}
\mathfrak{D}(f(T)) & =\left\{x \mid \lim _{n \rightarrow \infty} f\left(T \mid E\left(\delta_{n}\right) \mathfrak{X}\right) E\left(\delta_{n}\right) x \text { exists }\right\}, \\
f(T) x & =\lim _{n \rightarrow \infty} f\left(T \mid E\left(\delta_{n}\right) \mathfrak{X}\right) E\left(\delta_{n}\right) x, & x \in \mathfrak{D}(f(T)) .
\end{array}
$$

The operator $f(T)$ thus defined is a closed linear operator which is independent of the particular sequence $\left\{\delta_{n}\right\}$ of Borel sets used to define it. If $T$ is a closed scalar type operator the operational calculus may be extended from analytic functions to functions $f$ which are Borel measurable on the spectrum of $T$. For such an $f$ let $f_{n}$ be defined by the equations

$$
\begin{aligned}
& f_{n}(\lambda)=f(\lambda), \quad|f(\lambda)| \leqq n ; \\
& f_{n}(\lambda)=0, \quad|f(\lambda)|>n .
\end{aligned}
$$

Then the operator $f(T)$ may be defined by the equations

$$
\begin{aligned}
\mathfrak{D}(f(T)) & =\left\{x \mid \lim _{n} \int f_{n}(\lambda) E(d \lambda) x \text { exists }\right\}, & \\
f(T) & =\lim _{n} \int f_{n}(\lambda) E(d \lambda) x, & x \in \mathfrak{D}(f(T)) .
\end{aligned}
$$


The operator $f(T)$ thus defined is a closed operator with dense domain and, of course, coincides with the previously defined operator $f(T)$ in case $f$ is analytic. The usual rules for an operational calculus may be readily verified.

If $T$ is a closed scalar type spectral operator with spectral resolution $E$ and if $f$ is a Borel measurable function then the operator $f(T)$ is also a closed scalar type spectral operator whose spectral resolution $E_{1}$ is given by the formula $E_{1}(\delta)=E\left(f^{-1}(\delta)\right)$. In general if $T$ is an arbitrary closed spectral operator with spectral resolution $E$ and if $f$ is a single valued function, analytic on a domain $G$ which, when taken together with a finite number of exceptional points $p$ includes a neighborhood of $\sigma(T)$ and a neighborhood of the point at infinity, then $f(T)$ is a closed spectral operator provided that, at each exceptional point $p$, as well as at $\infty, f$ has at most a pole, and also $E(\{p\})$ $=0$ for each exceptional point $p$. In particular a polynomial function of a closed spectral operator is a closed spectral operator.

Bade $[3 ; 4]$ has developed, by means of some remarkably powerful arguments, a theory of algebras of spectral operators which extends many features of the theory of commutative $W^{*}$-algebras in Hilbert space. In particular he has given answers to the following questions. If $\tau$ is a family of commuting scalar type spectral operators, when are all the operators in the weakly closed algebra generated by $\tau$ also scalar type spectral operators, and precisely what are the operators in this weakly closed algebra generated by $\tau$ ? Before surveying this work of Bade on algebras of scalar type spectral operators I should like to describe briefly the structure of uniformly closed commutative algebras of general spectral operators. It will be convenient to use the term full algebra for a uniformly closed algebra of operators which contains the inverse of each of its nonsingular elements. The intersection of all the full algebras containing a given family of operators is called the full algebra generated by the family. A fundamental result in the study of algebras of spectral operators states that the uniformly closed algebra of operators generated by a bounded Boolean algebra of projections is a full algebra equivalent to the algebra of continuous functions on its own space of maximal ideals. From this it follows that if $\mathfrak{A}(\tau)$ is the full algebra generated by a family $\tau$ of commuting spectral operators in a $B$-space, $\mathfrak{X}$ together with their resolutions of the identity and if the Boolean algebra $\beta$ generated by the spectral measures of the operators in $\tau$ is bounded then $\mathscr{A}(\tau)$ is a vector direct sum

$$
\mathfrak{U}(\tau)=\mathfrak{A}(\beta)+\mathfrak{R}
$$


where $\Re$ is the radical in $\mathfrak{A}(\tau)$ and where $\mathfrak{A}(\beta)$ is the uniformly closed algebra generated by $\beta$. Furthermore $\mathfrak{A}(\beta)$ is equivalent to the algebra of continuous functions on the structure space $\Lambda$ of $\mathfrak{A}(\tau)$. This latter isomorphism may be represented by an integral of the form

$$
S^{*}(f)=\int_{\Lambda} f(\lambda) A(d \lambda), \quad f \in C(\Lambda),
$$

where $A$ is a spectral measure in $\mathfrak{X}^{*}$ and where $S^{*}(f)$ is the adjoint of the operator $S(f)$ in $\mathfrak{X}(\beta)$ corresponding to $f$. If the space $\mathfrak{X}$ is weakly complete then $A$ is the adjoint of a countably additive spectral measure $E$ in $\mathfrak{X}$ and the preceding formula takes the form

$$
S(f)=\int_{\Lambda} f(\lambda) E(d \lambda), \quad f \in C(\Lambda) .
$$

This type of formula raises the natural question of considering the operators it defines for bounded Borel functions $f$ which are not necessarily continuous. In this connection let us consider a countably additive spectral measure $E$ on a $\sigma$-field $\Sigma$ of subsets of a set $\Lambda$. Then a function $f$ on $\Lambda$ is said to be E-essentially bounded on $\Lambda$ if

$$
E-\operatorname{ess}_{\lambda \in \Lambda} \sup |f(\lambda)|=\inf _{E(\delta)=I} \sup _{\lambda \in \delta}|f(\lambda)|
$$

is finite. Since $E$ is countably additive on $\Sigma$ there is a set $\delta_{0}$ in $\Sigma$ with $E\left(\delta_{0}\right)=I$ and for which

$$
E-\underset{\lambda \in \Lambda}{\operatorname{ess} \sup }|f(\lambda)|=\sup _{\lambda \in \delta_{0}}|f(\lambda)| .
$$

The map $f \rightarrow S(f)$ defined by the integral $S(f)=\int_{\Delta} f(\lambda) E(d \lambda)$ is an isomorphism between the algebra $E B(\Lambda, \Sigma)$ of all $E$-essentially bounded $\Sigma$-measurable scalar functions on and a full algebra of scalar type spectral operators. The resolution of the identity $E(S(f))$ for $S(f)$ is given by the formula

$$
E(S(f), \sigma)=E\left(f^{-1}(\sigma)\right)
$$

where $\sigma$ is an arbitrary Borel set in the plane. The norm of $S(f)$ is related to that of $f$ by the inequalities

$$
E-\underset{\lambda \in \Lambda}{\operatorname{ess} \sup }|f(\lambda)| \leqq|S(f)| \leqq K E-\underset{\lambda \in \Delta}{\operatorname{esssup}}|f(\lambda)|,
$$

where $K$ is independent of $f$. It follows that $S(f)$ has a bounded inverse if and only if $f^{-1}$ is $E$-essentially bounded and that the spectrum of $S(f)$ is given by the formula 


$$
\sigma(S(f))=\bigcap_{E(\delta)=I} \overline{f(\delta),} \quad f \in E B(\Lambda, \Sigma) .
$$

Also if the sequence $\left\{f_{n}\right\}$ is bounded in $E B(\Lambda, \Sigma)$ and if $f_{n}(\lambda) \rightarrow f(\lambda)$ except for those $\lambda$ in a set of $E$-measure zero then $S\left(f_{n}\right) x \rightarrow S(f) x$ for every $x$ in $\mathfrak{X}$. Another corollary is that an algebra of operators in a weakly complete space which is topologically and algebraically equivalent to some algebra of bounded continuous functions consists entirely of scalar type operators. Thus every operator in the uniformly closed algebra generated by a bounded Boolean algebra of projections in a weakly complete space is a scalar type spectral operator. It follows that if the Boolean algebra generated by the spectral measures of the operators in a commutative family $\tau$ of spectral operators is bounded then every operator in the full algebra $\mathfrak{A}(\tau)$ generated by $\tau$ is a spectral operator. In Hilbert space the Boolean algebra generated by the spectral measures of a finite set $T_{1}, \cdots, T_{n}$ of commuting spectral operators is always bounded and so the full algebra $\mathfrak{A}$ generated by $T_{1}, \cdots, T_{n}$ and their spectral measures has the form $\mathfrak{A}=\mathfrak{B}+\mathfrak{R}$ where $\mathfrak{R}$ is the radical in $\mathfrak{A}$ and where $\mathfrak{B}$ consists of scalar type spectral operators and is the algebra generated by the spectral measures for $T_{1}, \cdots, T_{n}$. If $n=1$ this result is true for any $B$-space $\mathfrak{X}$ and furthermore every operator in $\mathfrak{B}$ is an $E$-essentially bounded function of the scalar part of $T_{1}$ ( $E$ is the spectral measure for $T_{1}$ ).

The preceding results concerning uniformly closed algebras are not difficult to prove and, for the most part may be found in [13]. We shall now describe Bade's results on strongly or weakly closed algebras of spectral operators. Since a convex set in the space of all bounded linear maps between two $B$-spaces has the same closure in the weak as in the strong operator topology the strong and weak operator closures of an algebra of operators are the same. Bade's basic result is the following theorem.

THEOREM (BADE). Let $\beta$ be a bounded Boolean algebra of projections in a weakly complete $B$-space $\mathfrak{X}$. Then the weakly closed operator algebra generated by $\beta$ consists of all operators in $\mathfrak{X}$ which leave invariant every closed linear manifold which is left invariant by every member of $\beta$.

One of the tools developed by Bade in his study is the following lemma which enables him to dispense with the scalar product in Hilbert space and thus work in more general $B$-spaces. The lemma concerns a $\sigma$-complete Boolean algebra $\mathfrak{B}$ of projections in a $B$-space $\mathfrak{X}$, and asserts that for each $x_{0}$ in $\mathfrak{X}$ there is a linear functional $x_{0}^{*}$ in $\mathfrak{X}^{*}$ with the properties that $x_{0}^{*} E x_{0} \geqq 0$ for $E$ in $\mathfrak{B}$ and also that if 
$x_{0}^{*} E x_{0}=0$ for some $E$ in $B$ then $E x_{0}=0$. A corollary of Bade's theorem is that every operator in the weakly closed algebra of operators in a weakly complete space which is generated by a bounded Boolean algebra $B$ of projections is a scalar type spectral operator. If in addition it is known that for some vector $x$ the set $\{E x \mid E \in \mathfrak{B}\}$ is a fundamental in $\mathfrak{X}$ then a bounded linear operator is in the weakly closed algebra generated by $B$ if and only if it commutes with every element of $\mathfrak{B}$. Another corollary is that every operator in the weakly closed operator algebra generated by a spectral operator of scalar type and its spectral measure is a spectral operator of scalar type.

Another result of Bade, which belongs to that interesting collection of principles dealing with conditions under which weak convergence implies strong convergence, is the following theorem.

If a generalized sequence of projections in a $\sigma$-complete Boolean algebra of projections converges weakly to a projection then it converges strongly. If the space is weakly complete then the assumption of $\sigma$ completeness may be replaced by that of boundedness. It has also been shown by Bade that if the Boolean algebra is complete then the weakly closed operator algebra it generates is the same as the uniformly closed algebra it generates.

Using some of the results on algebras of spectral operators Foguel [19] has proved the following perturbation theorem. Let $\left\{S_{n}\right\}$ be a sequence of commuting scalar type operators which converges strongly to the operator $S$. Let the Boolean algebra generated by the spectral measures of the operators $S_{n}$ be bounded. Then $S$ is a scalar type operator whose spectral measure $E$ is related to the spectral measure $E_{n}$ of $S_{n}$ by the equation $E(\sigma) x=\lim _{n} E_{n}(\sigma)$ for every $x$ in $\mathfrak{X}$ and Borel set $\sigma$ for which $E$ (boundary $\sigma$ ) $x=0$.

One feature of the theory of algebras of spectral operators which has, until recently, been neglected is the multiplicity theory. In 1956 Dieudonné [6] obtained such a theory under the assumption that the adjoint $\mathfrak{X}^{*}$ of the underlying space is separable. More recently Bade [4] has developed a new approach to multiplicity theory which does not require the separability assumption. We shall describe briefly Bade's theory. A cardinal valued function $m$ on a complete abstract Boolean algebra $\mathfrak{B}$ is called a multiplicity function if $m(0)=0$ and if $m\left(V E_{\alpha}\right)=\bigvee m\left(E_{\alpha}\right)$ for every set $\left\{E_{\alpha}\right\}$. The cardinal number $m(E)$ is called the multiplicity of $E$. The element $E$ is said to have uniform multiplicity $n$ if $m(F)=n$ whenever $0 \neq F \leqq E$. Any such multiplicity function $m$ on $\mathfrak{B}$ uniquely determines a family $\left\{E_{n}\right\}$, $n \leqq m(I)$, of disjoint elements of $\mathfrak{B}$ such that $I=\mathrm{V}_{n} E_{n}$ and $E_{n}$, if not 0 , has uniform multiplicity $n$. What is desired in case the Boolean 
algebra $\mathfrak{B}$ is a Boolean algebra of projections in the $B$-space $\mathfrak{X}$ is a multiplicity function which is a natural one in the sense that $m(E)$ is the least number of cyclic subspaces spanning the range of $E$. (The cyclic subspace $\mathfrak{M}(x)$ spanned by a vector $x$ is the closed linear manifold determined by all vectors of the form $E x, E \in \mathfrak{B}$.) If every family of disjoint projections in $\mathfrak{B}$ which is bounded by a given projection $E$ is at most countable then $E$ is said to satisfy the countable chain condition. Such $E$ form a dense $\sigma$-ideal in $\mathfrak{B}$ and what Bade does is to define $m(E)$ for such $E$ as the least cardinal number of cyclic subspaces spanning the range of $E$ and then prove that $m$ has a unique extension to a multiplicity function defined on all of $\mathfrak{B}$. Suppose that the identity $I$ in $\mathfrak{B}$ has a finite uniform multiplicity $n$ and that $\mathfrak{X}=\mathrm{V}_{i=1}^{n} \mathfrak{M}\left(x_{i}\right)$. Let us regard $\mathfrak{B}$ as a spectral measure on the Borel sets $\Sigma$ of its Stone space $\Omega$. Bade shows that there are functionals $x_{i}^{*}, i=1, \cdots, n$, with $x_{i}^{*} \mathfrak{M}\left(x_{j}\right)=0, j \neq i$, and that the measure $x_{i}^{*} E(\sigma) x_{i}=\mu_{i}(\sigma)$ is positive and dominates the vector measure $E(\sigma) x_{i}$ and uses these measures to define the direct $\operatorname{sum} L=\sum_{i=1}^{n} L_{1}\left(\Omega, \Sigma, \mu_{i}\right)$. The representation of $\mathfrak{X}$ and $\mathfrak{B}$ may now be stated as follows. There is a linear continuous one to one map $T$ of $\mathfrak{X}$ into a dense subspace of the direct sum $L$ such that if $T x=\left[f_{1}, \cdots, f_{n}\right]$ then

$$
\begin{aligned}
x_{i}^{*} E(e) x_{i} & =\int_{e} f_{i}(\omega) \mu_{i}(d \omega), \quad e \in \Sigma, i=1, \cdots, n, \\
x & =\lim _{m \rightarrow \infty} \sum_{i=1}^{n} \int_{e_{m}} f_{i}(\omega) E(d \omega) x_{i},
\end{aligned}
$$

where $e_{m}=\left\{\omega\left|f_{i}(\omega)\right| \leqq m, i=1, \cdots, n\right\}$. This is not as complete a description as one has for the well known Hilbert space case but, of course, it is not to be expected that $T$ maps $\mathfrak{X}$ onto all of $L$.

Bade proves that a projection $E$ in $\mathfrak{B}$ has finite uniform multiplicity $n$ if and only if its adjoint $E^{*}$ has finite uniform multiplicity $n$. As a corollary he obtains the important result that if $\mathfrak{X}$ is separable then $m(E)=m\left(E^{*}\right)$ for every $E$ in $\mathfrak{B}$. The main question left unanswered by Bade's work is the relation between $m(E)$ and $m\left(E^{*}\right)$ for projections of infinite multiplicity on nonseparable spaces.

Using the spectral representation associated with the spectral multiplicity theory of normal operators in Hilbert space and a result of Bade's, Foguel [18] has recently obtained some interesting results concerning the representation of operators in a separable Hilbert space $\mathfrak{S}$ which commute with a given bounded normal operator $S$ of finite multiplicity $n$ in $\mathfrak{W}$. In view of the classical spectral representation theory there is a finite decreasing sequence $e_{1} \supset e_{2} \supset \cdots \supset e_{n}$, of 
Borel sets in the complex plane with, $e_{1}$ the whole plane, and Borel measures $\mu_{j}$ with $\mu_{j}(e)=\mu\left(e e_{j}\right), j=1, \cdots, n$, such that $\mathfrak{S}$ is equivalent to the direct sum $\mathfrak{S}_{1}=\sum_{j=1}^{n} \oplus L_{2}\left(\mu_{j}\right)$. Under this equivalence the operator $S$ becomes the operator $S_{1}$ of multiplication, i.e., the operator which maps the vector $\left\{f_{1}(\lambda), \cdots, f_{n}(\lambda)\right\}$ in $\mathscr{S}_{1}$ into the vector $\left\{\lambda f_{1}(\lambda), \ldots, \lambda f_{n}(\lambda)\right\}$. Foguel's results are most clearly stated in terms of $S_{1}$ and $\mathfrak{S}_{1}$ and so we shall assume that $S$ is the operation of multiplication by the independent variable in the Hilbert space $\mathfrak{S}$ $=\sum_{i=1}^{n} \oplus L_{2}\left(\mu_{i}\right)$. Let $\mathfrak{A}$ be the algebra of bounded operators in $\mathfrak{S}$ which commute with $S$ and $\mathfrak{H}_{1}$ the algebra of $n \times n$ matrices $A(\lambda)$ $=\left(a_{i j}(\lambda)\right)$ of bounded measurable functions $a_{i j}$ for which $a_{i j}(\lambda)=0$ outside the set $e_{i} \cap e_{j}$. Then to each operator $A \in \mathfrak{A}$ corresponds a matrix $A(\lambda)$ which represents it in the sense that $A$ transforms the vector $\left[f_{1}, \cdots, f_{n}\right]$ into the vector $\left[\sum a_{i j}(\lambda) f_{j}(\lambda), \cdots, \sum a_{n j}(\lambda) f_{j}(\lambda)\right]$. This representation is unique (up to sets of measure zero) and the mapping $A \rightarrow A(\lambda)$ is a $*$-homomorphism. If $\mathfrak{Q}_{1}$ is normed by the expression $\max _{i, j}$ ess $\sup _{\lambda}\left|a_{i j}(\lambda)\right|$ then the mapping $A \rightarrow A(\lambda)$ is a homeomorphism. Strong convergence in $\mathfrak{A}$ is equivalent to uniform boundedness and convergence in measure (of the elements $a_{i j}(\lambda)$ ) in $\mathfrak{A}_{1}$. It follows as a corollary that if a sequence $\left\{A_{n}\right\}$ in $\mathfrak{A}$ converges strongly to the operator $A$ then $A_{n}^{*}$ converges strongly to $A^{*}$.

Foguel has also given an interesting canonical reduction for the matrices $A(\lambda)$. There are $n$ bounded measurable functions $z_{1}(\lambda), \cdots, z_{n}(\lambda)$, and $n$ disjoint projections $\epsilon_{1}(\lambda), \cdots, \epsilon_{n}(\lambda)$ whose elements are measurable and whose sum is the unit matrix, and a matrix $N(\lambda)$ which commutes with $\epsilon_{i}(\lambda)$, has measurable components, is nilpotent of order $n$, and for which

$$
A(\lambda)=\sum_{i=1}^{n} z_{i}(\lambda) \epsilon_{i}(\lambda)+N(\lambda)
$$

There is an increasing sequence $\left\{\alpha_{m}\right\}$ whose union is the complex plane and such that $A$ restricted to $E\left(\alpha_{m}\right) \mathfrak{S}$ ( $E$ is the resolution of the identity for $S$ ) is a spectral operator. Thus every operator $A$ in $\mathfrak{A}$ is the strong limit of a sequence of spectral operators. The operator $A$ will itself be a spectral operator if and only if the elements of the matrices $\epsilon_{1}, \cdots, \epsilon_{n}$ are almost everywhere bounded. It also follows that any generalized nilpotent in $\mathfrak{A}$ must be a nilpotent of order $n$.

The operator $A$ is compact if and only if there is a sequence $\left\{\lambda_{n}\right\}$ of eigenvalues for $S$ for which $A(\lambda)=0$ for almost all $\lambda$ not in the sequence $\left\{\lambda_{n}\right\}$ and $\lim _{n} A\left(\lambda_{n}\right)=0$. Consequently if $S$ has no eigenvalues then $A=0$ and if $S$ has only a finite number of eigenvalues then $A$ has a finite dimensional range. If $\lambda \notin\left\{\lambda_{n}\right\}$ then $\boldsymbol{z}_{i}(\lambda)=0, N(\lambda)=0$, 
$\epsilon_{1}(\lambda)=I$, and $\epsilon_{i}(\lambda)=0, i \geqq 2$.

Since every scalar type spectral operator in Hilbert space is equivalent to a normal operator the above results hold for the algebra $A$ of bounded operators which commute with a given bounded scalar type operator $S$ of finite multiplicity.

3. Conditions on the resolvent of an operator which are sufficient to insure that it is a spectral operator. In the preceding section it was observed that the resolvent of a spectral operator has the special properties (A), (B), (C) listed there, which are not properties of resolvents in general. In this section and the next it will be shown that these conditions and, in general, another condition on the space $\mathfrak{X}$ or the operator $T$ will prove to be sufficient to insure that $T$ is a spectral operator. In order to simplify the discussion we shall restrict ourselves to bounded operators. The central ideas to be developed in detail in $\$ \$ 3$ and 4 are in the literature ${ }^{9}$ but the treatment here is more general and at the same time more transparent since a number of unnecessary concepts have been eliminated from the earlier development and new proofs have replaced some of the original ones. This section will be divided into three parts. Part 3(A), will discuss consequences of the condition (A), Part 3(B), those of conditions (A) and (B), while Part $3(\mathrm{C})$ will discuss consequences of the conditions (A), (B), and (C). In these three parts it will be shown that an operator $T$ (in a weakly complete space) which has the properties (A), (B), and (C) has a uniquely determined countably additive spectral resolution defined on a $\sigma$-field $M(T)$ of sets measurable- $T$. In general this field need not contain all Borel sets and may not even contain enough sets to be useful at all. In Part 3(C) a fourth condition (D) is introduced so that the sets (A), . ., (D) are necessary as well as sufficient conditions for the operator $T$ to be a spectral operator.

The conditions (A), . . (D) are in abstract form and difficult to verify in most concrete problems. In order to make the analytical

9 These ideas were developed rather explicitly in [12] under the assumption that the spectrum is nowhere dense although in this work the present development was indicated. During the years 1953-1954 I had some correspondence with Gerhard Neubauer who made valuable suggestions concerning the work in [11] and [12]. In particular Neubauer observed that the single valued extension property of the resolvent was a necessary condition for the operator to be spectral. He also suggested a number of improvements that could be made in the theory presented in [12]. A summary of these ideas may be found in $[9 ; 10 ; 11]$. Also J. T. Schwartz has been a valuable critic and has made numerous contributions which have been incorporated in the presentation given in $\$ \$ 3$ and 4 . 
work of verification easier we will, in $\$ 4$, introduce a class of operators whose resolvents obey a growth condition $(G)$. This class is general enough to include most differential operators and restrictive enough so that the conditions (A), (C), and (D) may be verified. Thus, for a large class of operators the two conditions $(B)$ and $(G)$ may replace the four conditions (A), . , (D). For this reason the problem of verifying the boundedness condition (B) must be regarded as a key problem in applying the theory of spectral operators. The reader will readily perceive that $(B)$ is not the sort of condition that is likely to be easy to verify. This is not surprising, for what (B) amounts to in the final analysis is the assertion that the resolution of the identity is countably additive. Thus (B) is the condition which gives rise to the phenomenon of unconditional convergence of the eigenvalue expansions.

What we mean, in more detail, is this. It is because of the countable additivity of the resolution of the identity that spectral operators have important expansion theorems associated with them. For example, if $T$ is a spectral operator and its spectrum is denumerable, then every $x$ in $X$ has an unconditionally convergent expansion of the type $x=\sum x_{n}\left(=\sum_{\lambda \in \sigma(T)} E(\lambda) x\right)$ where the spectrum of $x_{n}$ consists of "generalized eigenvector" associated with $\lambda_{n}$. If $T$ is a spectral operator of scalar type, then the generalized eigenvectors are simply eigenvectors in the ordinary sense. If $T$ is a spectral operator of type $m$, then the generalized eigenvectors $x_{n}$ satisfy the equations $\left(\lambda_{n} I-T\right)^{m+1} x_{n}=0, n=1,2, \cdots$. Thus, as long as $T$ has a countably additive resolution of the identity, we are not far from the situation characteristic of normal operators in Hilbert space. If the countable additivity of the spectral resolution fails, so do many other convenient eigenvalue expansion properties.

It should be noted that it is by no means the case that all the familiar eigenvalue expansions of classical analysis are unconditionally convergent. Indeed, there are many examples, such as Fourier series expansions in the space $L_{p}(0,2 \pi)$ with $1<p<\infty$, where the expansion converges, but only conditionally. This seems to indicate that further developments in spectral theory will include a theory of conditionally convergent expansions associated with discrete and continuous spectra. Contributions along these lines have been made by D. R. Smart [36]. Nevertheless, the cases where one does have unconditionally convergent eigenvalue expansions are of sufficient importance to justify studying them for their own sake. It is this fact that lends importance to the problem of discovering which operators are spectral operators. 
3(A). Consequences of the condition (A). Here we shall be concerned with a bounded linear transformation $T$ in a complex $B$-space $\mathfrak{X}$. We shall establish a few properties of $T$ on the basis of the single assumption (A), which is repeated here for convenience of reference.

(A) For each $x$ in $\mathfrak{X}$ the function $R(\xi ; T) x$ has the single valued extension property.

Since $T$ satisfies condition (A) we recall that the resolvent set of $x$, which is denoted by $\rho(x)$, may be defined as the union of all the domains $D(f)$, the union being taken as $f$ varies over all analytic extensions of $R(\xi ; T) x$. Thus $\rho(x)$ is an open set containing $\rho(T)$, and its complement $\sigma(x)$ is a closed subset of $\sigma(T)$. The set $\sigma(x)$ is called the spectrum of $x$. It is clear that there is a unique maximal analytic extension of $R(\xi ; T) x$. This extension, which is a single valued analytic function defined on $\rho(x)$ will be denoted by $x(\cdot)$. Thus the function $x(\cdot)$ has, by definition, the properties

$$
\begin{aligned}
(\xi I-T) x(\xi) & =x, & & \xi \in \rho(x), \\
x(\xi) & =R(\xi ; T) x, & & \xi \in \rho(T) .
\end{aligned}
$$

Even though (A) is taken as a standing assumption throughout Part 3(A), it will be indicated parenthetically in the statement of each lemma in the proof of which it is used.

Lemma 1. (A) If $\alpha, \beta$ are complex numbers and $x, y$ are vectors in $\mathfrak{X}$, then

$$
\begin{aligned}
\sigma(x+y) & \subseteq \sigma(x) \cup \sigma(y), \\
\alpha x(\xi)+\beta y(\xi) & =(\alpha x+\beta y)(\xi), \quad \xi \in \rho(x) \rho(y) .
\end{aligned}
$$

Proof. The function $\alpha x(\xi)+\beta y(\xi)$ is an analytic extension of

$$
R(\xi ; T) \alpha x+R(\xi ; T) \beta y=R(\xi ; T)(\alpha x+\beta y), \quad \xi \in \rho(T),
$$

defined on the open set $\rho(x) \rho(y)$. Thus $\rho(\alpha x+\beta y) \supset \rho(x) \rho(y)$. For $\xi \in \rho(x) \rho(y)$ we have,

$$
(\alpha x+\beta y)(\xi)=\alpha x(\xi)+\beta y(\xi),
$$

by (A). Q.E.D.

Lemma 2. (A) The spectrum $\sigma(x)$ is void if and only if $x=0$.

Proof. If $\sigma(x)$ is void then $x(\xi)$ is an entire function. Since

$$
\lim _{\xi \rightarrow \infty} x^{*} x(\xi)=\lim _{\xi \rightarrow \infty} x^{*} R(\xi: T) x=0,
$$

it is seen that $x^{*} x(\xi)=0$ for all $\xi$ and all $x^{*}$ in the conjugate space $\mathfrak{X}^{*}$. Hence 


$$
x^{*} x=x^{*}(\xi I-T) x(\xi)=\left\{(\xi I-T)^{*} x^{*}\right\}(x(\xi))=0,
$$

for all $x^{*}$ in $\mathfrak{X}^{*}$, and thus it follows from the Hahn-Banach extension theorem that $x=0$. Q.E.D.

LEMma 3. (A) Let $\sigma$ be a set of complex numbers, and $\sigma^{\prime}$ its complement. If $x+y=x_{1}+y_{1}$, where $\sigma(x), \sigma\left(x_{1}\right) \subseteq \sigma$ and $\sigma(y), \sigma\left(y_{1}\right) \subseteq \sigma^{\prime}$, then $x=x_{1}, y=y_{1}$.

Proof. By Lemma 1

$$
\begin{aligned}
& \sigma\left(x-x_{1}\right) \subseteq \sigma(x) \cup \sigma\left(x_{1}\right), \\
& \sigma\left(y_{1}-y\right) \subseteq \sigma(y) \cup \sigma\left(y_{1}\right)
\end{aligned}
$$

and so the vector $x-x_{1}=y_{1}-y$ has a void spectrum. Thus Lemma 2 shows that $x=x_{1}, y=y_{1}$. Q.E.D.

Lemma 4. (A) If $P$ is a bounded linear operator in $\mathfrak{X}$ which commutes with $T$, then

$$
\sigma(P x) \subseteq \sigma(x), \quad x \in \mathfrak{X} .
$$

Proof. Since $P$ commutes with $T$ it commutes with the resolvent $R(\xi ; T)$ for every $\xi$ in $\rho(T)$. From the equation $R(\xi ; T) P x=P R(\xi ; T) x$ it is clear that $P x(\xi)$ is an analytic extension of $R(\xi ; T) P x$ to the domain $\rho(x)$. Thus $\rho(P x) \supseteq \rho(x)$ and hence $\sigma(P x) \subseteq \sigma(x)$. Q.E.D.

3(B). Consequences of the conditions (A) and (B). Throughout this section it will be assumed that the operator $T$ satisfies condition (A) of $3(\mathrm{~A})$ as well as the fundamental boundedness condition (B), which we restate here for convenience.

(B) There is a constant $K$, depending only upon $T$, such that for every pair $x, y$ of vectors with $\sigma(x), \sigma(y)$ disjoint we have

$$
|x| \leqq K|x+y| \text {. }
$$

Although the assumptions (A) and (B) will be standing assumptions throughout Part $3(\mathrm{~B})$, they will be indicated in the statement of each lemma where they are used.

Assumption (B) allows us to associate projections $E(\delta)$ with certain sets $\delta$ of complex numbers.

Definition 1. The symbol $S_{1}(T)$ will be used for the family of all sets $\sigma$ with the property that vectors of the form $x+y$ with $\sigma(x) \subseteq \sigma$, $\sigma(y) \subseteq \sigma^{\prime}$ are dense in $\mathfrak{X}$.

It is clear that if $\sigma$ is in $S_{1}(T)$, then the complement $\sigma^{\prime}$ is also in $S_{1}(T)$. 
Lemma 2. (A, B) If $\sigma$ is in $S_{1}(T)$, there is one and only one bounded projection $E(\sigma)$ on $\mathfrak{X}$ with the properties $E(\sigma) x=x$ if $\sigma(x) \subseteq \sigma$ and $E(\sigma) x=0$ if $\sigma(x) \subseteq \sigma^{\prime}$. Moreover,

$$
E(\sigma)+E\left(\sigma^{\prime}\right)=I, \quad E(\sigma) E\left(\sigma^{\prime}\right)=0, \quad|E(\sigma)| \leqq K .
$$

Proof. The properties

$$
E(\sigma) x=x \text { if } \sigma(x) \subseteq \sigma, \quad E(\sigma) x=0 \text { if } \sigma(x) \subseteq \sigma^{\prime},
$$

define the projection $E(\sigma)$ on the dense set $D=\{x+y \mid \sigma(x) \subseteq \sigma$, $\left.\sigma(y) \subseteq \sigma^{\prime}\right\}$. Thus the uniqueness of $E(\sigma)$ is assured by the requirement that it is bounded.

To prove that an $E(\sigma)$ with the properties (*) exists, note that by Lemma 3 of Part $3(\mathrm{~A})$ the properties $\left({ }^{*}\right)$ define a single-valued projection on $D$. Assumption (B) merely states that this projection is bounded, with bound at most $K$. Thus it has a unique extension by continuity to a projection with bound at most $K$ defined on $\mathfrak{X}$. Since it is clear that $\left(E(\sigma)+E\left(\sigma^{\prime}\right)\right) x=x$ and $E(\sigma) E\left(\sigma^{\prime}\right) x=0$ for $x$ in $D$, it follows by continuity that these properties hold for all $x$ in $\mathfrak{X}$. Q.E.D.

Lemma 3. (A, B) If $P$ is a bounded linear operator which commutes with $T$, then

$$
P E(\sigma)=E(\sigma) P, \quad \sigma \in S_{1}(T) .
$$

PRoof. For $\sigma$ in $S_{1}$, vectors of the form $z=x+y$ with $\sigma(x) \subseteq \sigma$ and $\sigma(y) \subseteq \sigma^{\prime}$ are dense in $\mathfrak{X}$. For such a vector $z$ we have $P z=P x+P y$ and, by Lemma 4 of Part 3(A), $\sigma(P x) \subseteq \sigma$ and $\sigma(P y) \subseteq \sigma^{\prime}$. Thus, by Lemma 2, $E(\sigma) P x=P x$ and $E(\sigma) P y=0$ and so by Lemma 2

$$
E(\sigma) P z=P x=P E(\sigma) z .
$$

Since the vectors $z$ are dense in $\mathfrak{X}$ we have $E(\sigma) P=P E(\sigma)$. Q.E.D.

We now introduce a subclass of $S_{1}(T)$, which will be shown to be a Boolean algebra.

Definition 4. (A, B) The symbol $S_{2}(T)$ will be used for the family of all sets $\sigma$ having the property that for every $x$ in $\mathfrak{X}$ and every $\epsilon>0$, there are vectors $x_{1}, x_{1}^{\prime}$ with $\sigma\left(x_{1}\right) \subseteq \sigma(x) \sigma, \sigma\left(x_{1}^{\prime}\right) \subseteq \sigma(x) \sigma^{\prime}$ and $\left|x_{1}+x_{1}^{\prime}-x\right|<\epsilon$.

It is clear that $S_{2}(T)$ is closed under complementation, and contains the void set and the whole plane.

Lemma 5. (A, B) The family $S_{2}(T)$ is a Boolean algebra.

Proof. Since $S_{2}(T)$ is closed under complementation, to prove the lemma it is sufficient to show that it contains the union of every pair of its elements. 
Let $\sigma_{1}$ and $\sigma_{2}$ be sets in $S_{2}(T)$ and, for every set $\mu$ of complex numbers, let

$$
\mathfrak{M}(\mu)=\{x \mid \sigma(x) \subseteq \mu\}
$$

for every subset $\mu$ of the complex plane. If $x$ is in $\mathfrak{X}$, then, since $\sigma_{1}$ is in $S_{2}(T), x$ is in the closure of $\mathfrak{M}\left(\sigma_{1} \sigma(x)\right)+\mathfrak{M}\left(\sigma_{1}^{\prime} \sigma(x)\right)$. On the other hand, it follows, since $\sigma_{2}$ is in $S_{2}(T)$, that $\mathfrak{M}\left(\sigma_{2} \sigma_{1}^{\prime} \sigma(x)\right)+\mathfrak{M}\left(\sigma_{2}^{\prime} \sigma_{1}^{\prime} \sigma(x)\right)$ is dense in $\mathfrak{M}\left(\sigma_{1}^{\prime} \sigma(x)\right)$. Since, by Lemma 1 of Part $3(\mathrm{~A}), \mathfrak{M}\left(\sigma_{1} \sigma(x)\right)$ $+\mathfrak{M}\left(\sigma_{2} \sigma_{1}^{\prime} \sigma(x)\right)$ is contained in $\mathfrak{M}\left(\left(\sigma_{1} \cup \sigma_{2}\right) \sigma(x)\right)$, it follows immediately that $x$ is in the closure of $\mathfrak{M}\left(\left(\sigma_{1} \cup \sigma_{2}\right) \sigma(x)\right)+\mathfrak{M}\left(\left(\sigma_{1} \cup \sigma_{2}\right)^{\prime} \sigma(x)\right)$. But this means that $\sigma_{1} \cup \sigma_{2}$ is in $S_{2}(T)$. Q.E.D.

Lемма 6. (A, B) The restriction of the projection valued function E from $S_{1}(T)$ to the Boolean algebra $S_{2}(T)$ is a spectral measure.

PROOF. The term spectral measure is used for a homomorphic map from a Boolean algebra into an algebra of projections provided that the units of the Boolean algebra map into the projection operators 0 and $I$ respectively. We use the notations of the proof of the preceding lemma and let $\sigma$ be in $S_{2}(T)$. Since an arbitrary vector is in the closure of $\mathfrak{M}(\sigma \sigma(x))+\mathfrak{M}\left(\sigma^{\prime} \sigma(x)\right)$ and since, by Lemma $2, E(\sigma)(z+y)=z$ for $z$ in $\mathfrak{M}(\sigma \sigma(x))$ and $y$ in $\mathfrak{M}\left(\sigma^{\prime} \sigma(x)\right)$, it follows that $E(\sigma) x$ is in $\mathrm{Cl} \mathfrak{M}(\sigma \sigma(x))$ (where the symbol $\mathrm{Cl} \mathfrak{M}(\sigma \sigma(x))$ is used for the closure of $\mathfrak{M}(\sigma \sigma(x)$ ), and that $E(\sigma) x=x$ for $x$ in $\mathrm{Cl} \mathfrak{M}(\sigma \sigma(x))$. Thus, if $\sigma_{1}, \sigma_{2}$ are in $S_{2}(T)$, then we have $E\left(\sigma_{1}\right) E\left(\sigma_{2}\right) x$ is in $\mathrm{Cl} \mathfrak{M}\left(\sigma_{1} \sigma_{2} \sigma(x)\right)$. Hence

$$
E\left(\sigma_{1} \sigma_{2}\right) E\left(\sigma_{1}\right) E\left(\sigma_{2}\right) x=E\left(\sigma_{1}\right) E\left(\sigma_{2}\right) x .
$$

Since

$$
E\left(\sigma_{1} \sigma_{2}\right) x \in \mathrm{Cl} \mathfrak{M}\left(\sigma_{1} \sigma_{2} \sigma(x)\right) \subseteq \mathrm{Cl} \mathfrak{M}\left(\sigma_{1} \sigma_{2}(x)\right) \cap \mathrm{Cl} \mathfrak{M}\left(\sigma_{2} \sigma(x)\right)
$$

we have

$$
E\left(\sigma_{1}\right) E\left(\sigma_{2}\right) E\left(\sigma_{1} \sigma_{2}\right) x=E\left(\sigma_{1} \sigma_{2}\right) x .
$$

Since all the projections $E(\cdot)$ commute with $T$ and hence with each other, by Lemma 3 , it follows that $E\left(\sigma_{1}\right) E\left(\sigma_{2}\right)=E\left(\sigma_{1} \sigma_{2}\right)$. Then we have

$$
\begin{aligned}
E\left(\sigma_{1}\right) \vee E\left(\sigma_{2}\right) & =E\left(\sigma_{1}\right)+E\left(\sigma_{2}\right)-E\left(\sigma_{1} \sigma_{2}\right) \\
& =I-\left(I-E\left(\sigma_{1}\right)\right)\left(I-E\left(\sigma_{2}\right)\right) \\
& =I-\left(E\left(\sigma_{1}^{\prime}\right) E\left(\sigma_{2}^{\prime}\right)\right)=I-E\left(\sigma_{1}^{\prime} \sigma_{2}^{\prime}\right) \\
& =E\left(\left(\sigma_{1}^{\prime} \sigma_{2}^{\prime}\right)^{\prime}\right)=E\left(\sigma_{1} \cup \sigma_{2}\right) .
\end{aligned}
$$

Definition 7. (A, B) The symbol $S(T)$ will be used for the collec- 
tion of those sets $\sigma$ in $S_{2}(T)$ for which there exist closed sets $\mu_{n}, \nu_{n}$ in $S_{2}(T)$ with $\mu_{n} \subseteq \sigma, \nu_{n} \subseteq \sigma^{\prime}, n=1,2, \cdots$ and

$$
x=\lim _{n \rightarrow \infty}\left[E\left(\nu_{n}\right)+E\left(\mu_{n}\right)\right] x, \quad x \in \mathfrak{X} .
$$

Lemma 8. (A, B) The family $S(T)$ is a Boolean algebra.

Proof. It is clear that $S(T)$ is closed under complementation. Hence, in order to show that $S(T)$ is a Boolean algebra, it will suffice to show that it is closed under the operation of forming unions.

Let $\sigma, \tilde{\sigma}$ be in $S(T)$. Let $\left\{\mu_{n}\right\}$ and $\left\{\nu_{n}\right\}$ be as in Definition 7 , and let $\left\{\tilde{\mu}_{n}\right\}$ and $\left\{\tilde{\nu}_{n}\right\}$ be sequences of closed sets in $S_{2}(T)$ such that $\tilde{\mu}_{n} \subseteq \tilde{\sigma}, \nu_{n} \subseteq \tilde{\sigma}^{\prime}$, and

$$
x=\lim _{n \rightarrow \infty}\left\{E\left(\tilde{\nu}_{n}\right) x+E\left(\tilde{\mu}_{n}\right) x\right\}, \quad x \in \mathfrak{X} .
$$

Since the sequence $\left\{E\left(\nu_{n}\right)+E\left(\mu_{n}\right)\right\}$ is strongly convergent it is bounded and the operators $E\left(\nu_{n}\right)+E\left(\mu_{n}\right)$ are therefore equi-continuous. Thus we have

$$
\begin{aligned}
x & =\lim _{n \rightarrow \infty}\left[E\left(\nu_{n}\right)+E\left(\mu_{n}\right)\right]\left[E\left(\tilde{\mu}_{n}\right)+E\left(\tilde{\nu}_{n}\right)\right] x \\
& =\lim _{n \rightarrow \infty}\left\{E\left(\mu_{n} \tilde{\mu}_{n} \cup_{\mu_{n} \tilde{\nu}_{n}} \cup \nu_{n} \tilde{\mu}_{n}\right) x+E\left(\nu_{n} \tilde{\nu}_{n}\right) x\right\}, \quad x \in \mathfrak{X} .
\end{aligned}
$$

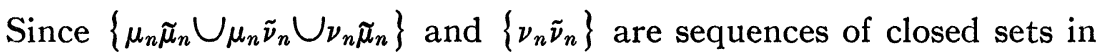
$S_{2}(T)$ contained in $\sigma \cup \tilde{\sigma}$ and $(\sigma \cup \tilde{\sigma})^{\prime}$ respectively, it follows that $\sigma \cup \tilde{\sigma}$ is in $S(T)$. Q.E.D.

Lemma 9. (A, B) The set $\sigma(T)$ belongs to $S(T)$, and $E(\sigma(T))=I$. Furthermore, every subset $\delta$ of the resolvent set $\rho(T)$ is in $S(T)$ and has $E(\delta)=0$.

Proof. Since $\sigma(x) \subseteq \sigma(T)$ for all $x$ in $\mathfrak{X}$, it is clear from Definition 4 and Lemma 2 that $\sigma(T)$ is in $S_{2}(T)$ and that $E(\sigma(T)) x=x$ for $x$ in $\mathfrak{X}$. Since $\sigma(T)$ is closed, it is clear from Definition 7 that $\sigma(T)$ is in $S(T)$. If $\delta \subseteq \rho(T)$ then the void set $\phi$ and the spectrum $\sigma(T)$ are closed subsets of $\delta, \delta^{\prime}$ respectively and $E(\phi) x+E(\sigma(T)) x=x$ which proves that $\delta$ is in $S(T)$. Since $E(\rho(T))=0$ we have $E(\delta)=E(\delta) E(\rho(T))=0$. Q.E.D.

LEMma 10. (A, B) Let $\left\{\sigma_{m}\right\}$ be a decreasing sequence of sets in $S(T)$ whose limit $\sigma$ is also in $S(T)$. Then

$$
E(\sigma) x=\lim _{m \rightarrow \infty} E\left(\sigma_{m}\right) x, \quad x \in \mathfrak{X} .
$$

Proof. We wish to show that $\lim _{m \rightarrow \infty} E\left(\sigma_{m}-\sigma\right) x=0$ for all $x$. Thus 
we may pass without loss of generality from consideration of the sequence $\left\{\sigma_{m}\right\}$ to consideration of the sequence $\left\{\sigma_{m}-\sigma\right\}$; that is, we may and shall assume without loss of generality that $\sigma$ is void. Since $E\left(\sigma_{m}\right)=E\left(\sigma_{m} \sigma(T)\right)$, by the preceding lemma, we may also assume that $\sigma_{m} \subseteq \sigma(T)$.

Suppose that our assertion is false, so that there is a $p>0$ and a vector $x$ such that $\left|E\left(\sigma_{m}\right) x\right| \geqq p$ for arbitrarily large $m$. Passing without loss of generality to a subsequence, we may assume that $\left|E\left(\sigma_{m}\right) x\right|$ $\geqq p$ for all $m$.

For $\sigma$ in $S_{2}(T)$ let $M(\sigma)=\sup _{\mu} \subseteq \sigma|E(\mu) x|, \mu \in S_{2}(T)$. It is clear that if $\nu_{1} \subseteq \nu_{2}$ then $M\left(\nu_{1}\right) \leqq M\left(\nu_{2}\right)$. Let $\mu \subseteq \nu_{1} \cup \nu_{2}$. Since $|E(\mu) x|$ $=\left|E\left(\mu \nu_{1}\right) x+E\left(\mu \nu_{1}^{\prime} \nu_{2}\right) x\right| \leqq M\left(\nu_{1}\right)+M\left(\nu_{2}\right)$, it follows immediately that

$$
M\left(\nu_{1} \cup \nu_{2}\right) \leqq M\left(\nu_{1}\right)+M\left(\nu_{2}\right) \text {. }
$$

Since

$$
E(\mu) x=|E(\mu) E(\sigma) x| \leqq K|E(\sigma) x|
$$

for $\mu \subseteq \sigma$ it is seen that

$$
M(\sigma) \leqq K|E(\sigma) x|
$$

Since $\sigma_{m}$ is in $S(T)$, we can find closed sets $\mu_{m}$ and $\nu_{m}$ in $S_{2}(T)$ such that $\mu_{m} \subseteq \sigma_{m}, \nu_{m} \subseteq \sigma_{m}^{\prime}$, and

$$
\left|E\left(\mu_{m} \cup \nu_{m}\right)^{\prime} x\right| \leqq p K^{-1} 2^{-m-1} .
$$

Then

$$
M\left(\left(\mu_{m} \cup \nu_{m}\right)^{\prime}\right) \leqq p 2^{-m-1},
$$

so that, putting $\delta_{m}=\sigma_{m}-\mu_{m}$, we have $\delta_{m}=\sigma_{m} \mu_{m}^{\prime} \subseteq \mu_{m}^{\prime} \cap \nu_{m}^{\prime}=\left(\mu_{m} \cup \nu_{m}\right)^{\prime}$ and so

$$
M\left(\delta_{m}\right) \leqq p 2^{-m-1} .
$$

It follows that no finite sum $\delta_{1} \cup \cdots \cup \delta_{n}$ can cover $\sigma_{n}$. Indeed,

$$
M\left(\delta_{1} \cup \cdots \cup \delta_{n}\right) \leqq \sum_{i=1}^{n} p 2^{-i-1} \leqq 1 / 2^{p}
$$

while $\left|E\left(\sigma_{n}\right) x\right| \geqq p$. Hence

$$
\sigma_{n} \mu_{1} \mu_{2} \cdots \mu_{n}=\sigma_{n}-\bigcup_{i=1}^{n} \sigma_{n} \delta_{i}
$$

is nonvoid. Since $\bigcap_{i=1}^{n} \mu_{i}$ is a decreasing sequence of nonvoid closed subsets of the compact set $\sigma(T)$, we have $\bigcap_{i=1}^{n} \mu_{i} \neq \phi$. Thus, since 
$\mu_{i} \subseteq \sigma_{i}$ it follows that $\bigcap_{i=1}^{n} \sigma_{i} \neq \phi$, contrary to assumption. Q.E.D.

The following three theorems summarize the results of this section and at the same time lay the foundation for the studies to be made in $\$ 4$ that follows.

Theorem 11. (A, B) Let $T$ be a bounded linear operator in the complex $B$-space $\mathfrak{X}$. Then there is a unique spectral measure on the field $S(T)$ with the properties

$$
\begin{aligned}
& E(\delta) x=x, \quad \delta \in S(T), \quad \sigma(x) \subseteq \delta, \\
& =0 . \quad \delta \in S(T), \quad \sigma(x) \subseteq \delta^{\prime} .
\end{aligned}
$$

This spectral measure is bounded, countably additive on $S(T)$, and commutes with $T$.

Proof. From Definitions 1,4 and 7 it is seen that $S(T) \subseteq S_{1}(T)$, and thus for each $\delta$ in $S(T)$ there is, by Lemma 2, one and only one projection $E(\delta)$ with $E(\delta) x=x$ if $\sigma(x) \subseteq \delta$ and $E(\delta) x=0$ if $\sigma(x) \subseteq \delta^{\prime}$. Lemma 2 also shows that $|E(\delta)|$ is bounded in $\delta$. Lemma 3 shows that $E(\delta)$ commutes with $T$ and Lemmas 6,8 and 10 show that $E$ is a countably additive spectral measure on $S(T)$. Q.E.D.

Since the field $S(T)$ is not necessarily a $\sigma$-field it is natural to ask whether or not the spectral measure $E$ may be extended to the $\sigma$ field generated by $S(T)$. The following definition and theorems are concerned with this question.

Definition 12. The symbol $M(T)$ will be used for the $\sigma$-complete Boolean algebra (or $\sigma$-field) determined by the Boolean algebra $S(T)$. The sets in $M(T)$ are called sets measurable $T$ or $T$-measurable sets.

Theorem 13. (A, B) Let $T$ be a bounded linear operator in the complex $B$-space $\mathfrak{X}$ and let $E$ be the associated spectral measure whose existence was established in Theorem 11. Then, in the conjugate space $\mathfrak{X}^{*}$, there is a unique extension of the adjoint $E^{*}$ to a spectral measure on the $\sigma$-field $M(T)$ of sets measurable $T$ which is countably additive on $M(T)$ in the $\mathfrak{X}$ topology of $\mathfrak{X}^{*}$. This unique extension is bounded and commutes with $T^{*}$.

Proof. For every $x$ in $\mathfrak{X}$ and $x^{*}$ in $\mathfrak{X}^{*}$ there is, according to the Hahn theorem, a unique countably additive extension $m\left(e, x, x^{*}\right)$ of the $x^{*} E(e) x$ from $S(T)$ to $M(T)$. From its uniqueness it is seen that $m\left(e, x, x^{*}\right)$ is bilinear in $x, x^{*}$ and from the boundedness of $|E(e)|$ follows the boundedness of $m\left(e, x, x^{*}\right)$. Thus for each $e$ in $M(T)$ there is a uniquely defined bounded linear operator $A(e)$ in $\mathfrak{X}^{*}$ for which

$$
x A(e) x^{*}=m\left(e, x, x^{*}\right) .
$$


It will next be shown that the mapping $e \rightarrow A(e)$ of $M(T) \rightarrow B\left(\mathfrak{X}^{*}\right)$ is a spectral measure. It clearly preserves finite disjoint unions, takes complements into complements, is countably additive in the $\mathfrak{X}$ topology of $\mathfrak{X}^{*}$, and is bounded. It remains only to show that

$$
A(\sigma) A(\delta)=A(\sigma \delta) \text {. }
$$

It is seen, by using the above remarks, that for a fixed $\sigma$ the family of $\delta$ for which the equation is valid is a $\sigma$-field. Thus if $\sigma$ is in $S(T)$ the equation holds for all $\delta$ in $M(T)$. Analogously, if $\delta$ is fixed in $M(T)$, then since the equation holds for $\sigma$ in a $\sigma$-field containing $S(T)$, it must hold for all $\sigma$ in $M(T)$. have

Since $T$ and $E(\delta)$ commute and since $A(\delta)=E(\delta) *$ for $\delta$ in $S(T)$ we

$$
x T^{*} A(\delta) x^{*}=x A(\delta) T^{*} x^{*}, \quad x \in \mathfrak{X}, x^{*} \in \mathfrak{X}^{*}, \delta \in S(T) .
$$

Since $A$ is countably additive in the $\mathfrak{X}$ topology of $\mathfrak{X}^{*}$ this identity holds for every $\delta$ in the $\sigma$-field determined by $S(T)$ and this proves that $A(\delta)$ commutes with $T^{*}$ for every $T$-measurable set $\delta$. Since $m\left(e, x, x^{*}\right)=x^{*} A(e) x$ is bounded in $e$ it follows from the principle of uniform boundedness that $|A(e)|$ is bounded for $e$ in $M(T)$. Q.E.D.

TheOREM 14. (A, B) Let $T$ be a bounded linear operator in the weakly complete complex $B$-space $\mathfrak{X}$ and let $E$ be the associated spectral measure whose existence was established in Theorem 11. Then there is a uniquely determined extension of $E$ to a spectral measure on the $\sigma$-field $M(T)$ of sets measurable $T$ which is countably additive on $M(T)$ in the strong operator topology. This extension is bounded and commutes with $T$.

Proof. Let $A$ be the spectral measure in the adjoint space $\mathfrak{X}^{*}$ which is associated with $T^{*}$ as in the preceding theorem. If $\mathfrak{X}$ is weakly complete, $A(\delta)$ is the adjoint of an operator $E(\delta)$ in $\mathfrak{X}$. To see this, note that the family of all sets $\delta$ for which there exists an operator $E(\delta)$ in $\mathfrak{X}$ with $A(\delta)=E(\delta)$ * contains $S(T)$. This family is also a Boolean algebra since $A$ is a spectral measure. Since $\mathfrak{X}$ is weakly complete it is a $\sigma$-complete Boolean algebra and hence coincides with $M(T)$. Since $E^{*}(\delta)$ commutes with $T^{*}$ it follows that $E(\delta)$ commutes with $T$ for every $\delta$ in $M(T)$. Theorem 13, together with the OrliczPettis theorem, shows that $E$ is countably additive on $M(T)$ in the strong operator topology. The boundedness of $E$ follows from that of A. Q.E.D.

$3(\mathrm{C})$. Consequences of the conditions $(A, B, C)$ : necessary and sufficient conditions for spectral operators. Theorem 14 of Part 3(B) falls short of proving that $T$ is a spectral operator in two ways. First 
of all the spectral measure $E$ is not necessarily a resolution of the identity for $T$, for, even though it commutes with $T$ it may not satisfy the inclusion relation $\sigma(T \mid E(\delta) \mathfrak{X}) \subseteq \bar{\delta}$. Secondly, the field $S(T)$ or the $\sigma$-field $M(T)$ may not contain ill Borel sets. The first of these difficulties will be eliminated by the hypothesis (C) to be made presently. The second of these difficulties leads us to consider operators which are spectral relative to a field other than the field of Borel sets. Such operators are described as follows:

Definition 1. Let $\Sigma$ be a field of sets in the complex plane and let $T$ be a linear operator in the complex $B$-space $\mathfrak{X}$. Then a spectral measure $E$ on $\Sigma$ is said to be a resolution of the identity for $T$ if it commutes with $T$ and satisfies

$$
\sigma(T \mid E(\delta) \mathfrak{X}) \subseteq \bar{\delta}, \quad \delta \in \Sigma,
$$

where $T \mid E(\delta) \mathfrak{X}$ is the restriction of $T$ to $E(\delta) \mathfrak{X}$. The operator $T$ is said to be a spectral operator of class $\left(\Sigma, \mathfrak{X}^{*}\right)$ if it has a bounded resolution of the identity on $\Sigma$ for which the set functions $x^{*} E(\cdot) x$ with $x$ in $\mathfrak{X}$ and $x^{*}$ in $\mathfrak{X}^{*}$ are all countably additive on $\Sigma$. An operator in $\mathfrak{X}^{*}$ is said to be a spectral operator of class $(\Sigma, \mathfrak{X})$ if it has a bounded resolution of the identity $A$ on $\Sigma$ for which the set functions $x A(\cdot) x^{*}$ with $x$ in $\mathfrak{X}$ and $x^{*}$ in $\mathfrak{X}^{*}$ are all countably additive on $\Sigma$.

Thus $T$ is a spectral operator if and only if it is a spectral operator of class $\left(B, \mathfrak{X}^{*}\right)$, where $B$ is the field of Borel sets in the plane.

Besides the conditions (A) and (B) the following condition (C) will be assumed in most of what follows. However, when any of the assumptions (A), (B), (C) are made in a lemma or theorem they will be indicated parenthetically.

(C) For every closed set $\delta$ of complex numbers the set of all vectors $x$ with $\sigma(x) \subseteq \delta$ is also closed.

Lemma 2. (A, B, C) For every set $\delta$ in $S_{1}(T)$ and every vector $z$ in $\mathfrak{X}$ we have $\sigma(E(\delta) z) \subseteq \bar{\delta} \sigma(z)$.

Proof. Since $\delta$ is in $S_{1}(T)$, an arbitrary vector $z$ in $\mathfrak{X}$ is the limit of a sequence $z_{n}=x_{n}+y_{n}$ with $\sigma\left(x_{n}\right) \subseteq \delta$ and $\sigma\left(y_{n}\right) \subseteq \delta^{\prime}$. Thus

$$
\sigma\left(E(\delta) z_{n}\right)=\sigma\left(x_{n}\right) \subseteq \bar{\delta}
$$

and since $E(\delta) z_{n} \rightarrow E(\delta) z$, it follows from (C) that

$$
\sigma(E)(\delta) z) \subseteq \bar{\delta} .
$$

Since $E(\delta)$ commutes with $T$ (cf. Lemma 3 of Part 3(B)), it is seen from Lemma 4 of Part (A) that $\sigma(E(\delta) z) \subseteq \sigma(z)$. Thus $\sigma(E(\delta) z)$ $\subseteq \bar{\delta} \sigma(z)$. Q.E.D. 
Lemma 3. (A, B, C) For $\delta$ in $S_{1}(T)$ let $T \mid E(\delta) \mathfrak{X}$ be the restriction of $T$ to $E(\delta) \mathfrak{X}$. Then

$$
\sigma(T \mid E(\delta) \mathfrak{X}) \subseteq \bar{\delta}, \quad \delta \in S_{1}(T) .
$$

Proof. It follows from Lemma 3 of Part 3(B) that $T$ commutes with $E(\delta)$ and so $T$ maps $E(\delta) \mathfrak{X}$ into itself. It is therefore meaningful to speak of the spectrum of the restriction of $T$ to $E(\delta) X$.

Let $\xi \notin \bar{\delta}$. It will first be shown that $\xi I-T$ is one-to-one on $E(\delta) \mathfrak{X}$. If $x$ is in $E(\delta) X$ and $(\xi I-T) x=0$, then, since

$$
R(\lambda ; T)=\sum_{n=0}^{\infty} \frac{(T-\xi I)^{n}}{(\lambda-\xi)^{n+1}},
$$

for all large $\lambda$, it is seen that $x(\lambda)=x /(\lambda-\xi)$ for $\lambda \neq \xi$. Thus the spectrum $\sigma(x)$ contains at most the point $\xi$ and therefore $\bar{\delta} \sigma(x)$ is void. Since $x=E(\delta) x$ it follows from Lemma 2 that $\sigma(x)$ is void and from Lemma 2 of Part 3(A) that $x=0$. This shows that $\xi I-T$ is one-to-one on $E(\delta) \mathfrak{X}$.

It will be shown that $(\xi I-T) E(\delta) \mathfrak{X}=E(\delta) \mathfrak{X}$. Let $x=E(\delta) x$ be an arbitrary point in $E(\delta) \mathfrak{X}$. Then, by Lemma $2, \sigma(x) \subseteq \bar{\delta}$ and so $\xi \in \rho(x)$. Thus $(\xi I-T) x(\xi)=x$ and hence $(\xi I-T) E(\delta) x(\xi)=E(\delta) x=x$ which shows that $(\xi I-T) E(\delta) \mathfrak{X}=E(\delta) \mathfrak{X}$. The operator $\xi I-T$ therefore maps $E(\delta) \mathfrak{X}$, in a one-to-one manner, onto all of itself. This means that $\xi$ is in $\rho(T \mid E(\delta) \mathfrak{X})$ and thus $\sigma(T \mid E(\delta) \mathfrak{X}) \subseteq \bar{\delta}$. Q.E.D.

TheOREM 4. A spectral operator $T$ has the properties (A), (B), and (C). Conversely, if the bounded linear operator $T$ has these properties it is a spectral operator of class $\left(S(T), \mathfrak{X}^{*}\right)$. Moreover, $T$ has a resolution of the identity which is countably additive in the strong operator topology.

Proof. We shall only demonstrate here the sufficiency of the conditions. Let the bounded linear operator $T$ satisfy the conditions (A), (B), and (C). Then by Theorem 11 of Part 3(B) and Lemma 3, $T$ is a spectral operator of class $\left(S(T), \mathfrak{X}^{*}\right)$ with a resolution of the identity which is countably additive in the strong operator topology. Q.E.D.

THEOREM 5. Let $T$ be a bounded linear operator in a weakly complete space. Then $T$ is a spectral operator if and only if $T$ satisfies conditions (A), (B), (C), and the following condition (D):

(D) Every complex number is interior to a set of arbitrarily small diameter belonging to $S(T)$.

Proof. It follows from the preceding theorem that a spectral operator has properties (A) through (C). To show that a spectral oper- 
ator $T$ has property (D), let $\delta$ be a closed set in the complex plane, and let $\left\{\delta_{n}\right\}$ be an increasing sequence of closed sets whose union is the complement $\delta^{\prime}$ of $\delta$. Let $E$ be the spectral resolution of $T$. Then

$$
x=\lim _{n}\left\{E(\delta) x+E\left(\delta_{n}\right) x\right\} .
$$

But $\sigma(E(\delta) x) \subseteq \delta$ and $\sigma\left(E\left(\delta_{n}\right) x\right) \subseteq \delta_{n}$. This shows that $\delta$ is in $S_{1}(T)$. Lemma 2 shows that $\delta$ is in $S_{2}(T)$ and, since $\delta_{n}$ is closed, the above equation proves that $\delta$ is in $S(T)$. Thus $S(T)$ contains every closed set and property (D) is evident.

Conversely, if the operator $T$ satisfies conditions (A) through (D), then, by Theorem 4 , it is a spectral operator of class $\left(S(T), \mathfrak{X}^{*}\right)$. According to Theorem 14 of Part 3(B) the resolution of the identity for $T$ has a unique extension to a countably additive spectral measure $E$ on $M(T)$. It will next be shown that $M(T)$ contains all Borel sets.

To do this let $U$ be an open set of the complex plane, and let $K$ be a compact subset of $U$. Then, by (D), each point $p$ in $K$ is interior to a certain set $\sigma_{p}$ in $S(T)$ with $\sigma_{p} \subseteq U$. Since $K$ is compact, it is contained in the union $\sigma$ of a finite collection of the sets $\sigma_{p}$. Thus, we have shown that if $K$ is a compact subset of $U$, there exists a set $\sigma \in S(T)$ such that $K \subseteq \sigma \subseteq U$. Since $U$ is the union of a countable infinity of its own compact subsets, it follows $U$ is in $M(T)$. Since $M(T)$ contains all open sets, it contains the family $B$ of all Borel sets.

To complete the proof it will suffice to show that $\sigma(T \mid E(\delta) \mathfrak{X}) \subseteq \bar{\delta}$ for every Borel set $\delta$. If $\lambda \notin \bar{\delta}$, then, using (D), the compact set $\bar{\delta} \sigma(T)$ may be covered by a set $\sigma$ in the field $S(T)$ with $\lambda \notin \bar{\sigma}$. Since $T$ is a spectral operator of class $\left(S(T), \mathfrak{X}^{*}\right)$, we have $\sigma(T \mid E(\sigma) \mathfrak{X}) \subseteq \bar{\sigma}$ and consequently $\lambda$ is in $\rho(T \mid E(\sigma) \mathfrak{X})$ which means that $\lambda I-T$ is a one-toone map of $E(\sigma) \mathfrak{X}$ into all of itself. Since $\sigma \supseteq \delta \sigma(T)$, we have $E(\sigma)$ $\supseteq E(\delta \sigma(T))=E(\delta)$ and consequently $E(\delta) \mathfrak{X}$ is an invariant subspace of $E(\sigma) \mathfrak{X}$. Thus $\lambda I-T$ is a one-to-one map of $E(\delta) \mathfrak{X}$ into all of itself. This proves that $\lambda$ is in $\rho(T \mid E(\delta) \mathfrak{X})$ and thus that $\sigma(T \mid E(\delta) \mathfrak{X}) \subseteq \bar{\delta}$. Q.E.D.

We conclude this section with two results on adjoint operators.

Lemma 6. Let $\Sigma$ be a field of sets in the complex plane and let $T$ be a spectral operator of class $\left(\Sigma, \mathfrak{X}^{*}\right)$. Then its adjoint $T^{*}$ is a spectral operator of class $(\Sigma, \mathfrak{X})$.

Proof. Let $E$ be a resolution of the identity for $T$. Then the mapping $\sigma \rightarrow E^{*}(\sigma)$ of $\Sigma$ into $B\left(\mathfrak{X}^{*}\right)$ is a spectral measure in $\mathfrak{X}^{*}$. Moreover, $x E^{*}(\sigma) x^{*}$ is evidently countably additive on $\Sigma$ for each $x \in \mathfrak{X}$ and $x^{*} \in \mathfrak{X}^{*}$. Let $\lambda \notin \bar{\sigma}$. Then the restriction of $\lambda I-T$ to $E(\sigma) \mathfrak{X}$ has an 
inverse $R_{\sigma}$. Define the operator $P_{\sigma}$ in $\mathfrak{X}$ by putting $P_{\sigma}=R_{\sigma} E(\sigma)$. Then clearly $E(\sigma) P_{\sigma}=P_{\sigma}=P_{\sigma} E(\sigma)$. Hence

$$
P_{\sigma}^{*} E(\sigma)^{*}=E(\sigma)^{*} P_{\sigma}^{*},
$$

so that $P_{\sigma}^{*}$ maps $E(\sigma) * \mathfrak{X}^{*}$ into itself. Also $(\lambda I-T) P_{\sigma}=E(\sigma)$, and $P_{\sigma}(\lambda I-T)=P_{\sigma} E_{\sigma}(\lambda I-T)=P_{\sigma}(\lambda I-T) E(\sigma)=E(\sigma)$. Thus

$$
P_{\sigma}^{*}\left(\lambda I^{*}-T^{*}\right)=\left(\lambda I^{*}-T^{*}\right) P_{\sigma}^{*}=E(\sigma)^{*} \text {. }
$$

Consequently the restriction of $P_{\sigma}^{*}$ to $E(\sigma) * \mathfrak{X}^{*}$ is the inverse of the restriction of $\lambda I^{*}-T^{*}$ to $E(\sigma) * \mathfrak{X}^{*}$. Hence $\lambda$ is in the resolvent of the restriction $\left(T^{*}\right)_{\sigma}$ of $T^{*}$ to $E^{*}(\sigma) \mathfrak{X}^{*}$. This shows that $\sigma\left(\left(T^{*}\right)_{\sigma}\right) \subseteq \bar{\sigma}$ and completes the proof. Q.E.D.

Theorem 7. (A, B, C, D) Let $T$ be a bounded linear operator in the complex $B$-space $\mathfrak{X}$ and let $B$ be the field of Borel sets in the plane. Then $T^{*}$ is a spectral operator of class $(B, \mathfrak{X})$.

Proof. In view of condition (D) we have $B \subseteq M(T)$. By Theorem 13 of Part $3(\mathrm{~B})$ the spectral measure $E^{*}$ of the preceding lemma may be extended from $S(T)$ to a spectral measure defined on $M(T)$. Then, as in the proof of Theorem 5 , it may be shown that $\sigma(T \mid E(\delta) \mathfrak{X}) \subseteq \bar{\delta}$ for each $\delta$ in $M(T)$. Q.E.D.

4. Operators whose spectra lie in a Jordan curve. In the preceding section it was seen that operators satisfying the conditions (A), $\cdots$, (D) are spectral operators. In this section it will be shown that, in certain important special cases, all of these conditions, except possibly the boundedness condition (B), are automatically satisfied. Thus for the special types of operators, condition (B) becomes the condition which is necessary as well as sufficient for the operator to be a spectral operator.

LEMмA 1. The condition (A) is satisfied if the spectrum of $T$ is nowhere dense in the complex plane.

Proof. If the resolvent set is dense then any two analytic, or even continuous, extensions of $R(\lambda ; T) x$ must coincide on their common domain of continuity. Q.E.D.

All of the special type operators to be considered in the present section will have nowhere dense spectra so that, according to Lemma 1 , the condition (A) will be satisfied by all of the operators that will be studied here.

The following theorem, which applies in particular to compact 
operators, gives a topological restriction on the spectrum of $T$ which guarantees that (A), (C), and (D) are all satisfied.

THEOREM 2. If the spectrum of an operator in a weakly complete space is totally disconnected then it is a spectral operator if and only if the boundedness condition (B) is satisfied.

Proof. Let $T$ be a bounded linear operator in the weakly complete $B$-space $\mathfrak{X}$. To prove the theorem it will, in view of Theorem 5 of Part 3(C), suffice to show that $T$ has the properties (A), (C), and (D). Since the spectrum $\sigma(T)$ of $T$ is totally disconnected it is nowhere dense and, according to Lemma 1 , condition (A) is satisfied.

We recall ${ }^{10}$ that to each spectral set (a spectral set is one which is an open and closed subset of the spectrum $\sigma(T)$ in its relative topology) is associated a projection $E(\delta)$ with $\sigma(T \mid E(\delta) \mathfrak{X})=\delta$ from which it follows that every spectral set is in $S_{2}(T)$. Since spectral sets are closed they are also contained in $S(T)$. Since the spectrum is totally disconnected, every spectral point is contained in a spectral set of arbitrarily small diameter and thus in an $S(T)$ set of arbitrarily small diameter. Since it is clear that every subset of the resolvent set is an $S(T)$ set, condition (D) is immediate.

To verify condition (C), let $\delta$ be a closed set of complex numbers and let

$$
M(\delta)=\{x \mid \sigma(x) \subseteq \delta\} .
$$

Condition (C) will be proved by showing that $M(\delta)$ is closed. Since $\sigma(x) \subseteq \sigma(T)$ we have $M(\delta)=M(\delta \sigma(T))$, and it may therefore be assumed, without loss of generality, that $\delta \subseteq \sigma(T)$. Since $\sigma(T)$ is totally disconnected, the closed set $\delta$ is an intersection $\bigcap_{\alpha} \delta_{\alpha}$ of spectral sets $\delta_{\alpha}$. Now clearly

$$
M(\delta)=M\left(\bigcap_{\alpha} \delta_{\alpha}\right)=\bigcap_{\alpha} M\left(\delta_{\alpha}\right),
$$

and so to see that $M(\delta)$ is closed it will suffice to see that $M\left(\delta_{\alpha}\right)$ is closed. Since $\delta_{\alpha}$ is a spectral set, it follows that $M\left(\delta_{\alpha}\right)=E\left(\delta_{\alpha}\right) \mathfrak{X}$ and hence is closed. Q.E.D.

Theorem 2 suggests that the difficulties which may be encountered in verifying the conditions (C) and (D) are, in some way, related to the presence of connected components of the spectrum. The remainder of the present section will be devoted to a study of the case where the spectrum is contained in a finite disjoint union of con-

10 Here we are using the operational calculus on analytic functions as developed in $[7]$ and $[8]$. 
nected sets each one of which is a Jordan arc. Before passing to the details of this study the following result will be introduced to allow us, without any loss of generality, to study the case where the whole spectrum is contained in one Jordan arc.

Theorem 3. Let $T$ be an operator in the B-space $\mathfrak{X}$. If $\mathfrak{X}$ is the direct sum of two of its closed subspaces $\mathfrak{X}_{1}$ and $\mathfrak{X}_{2}$, each invariant under $T$, and if the restrictions of $T$ to $\mathfrak{X}_{1}$ and $\mathfrak{X}_{2}$ are both spectral operators then $T$ is a spectral operator.

PROoF. Let $E_{1}$ and $E_{2}$ be the spectral resolutions of the restrictions of $T$ to $\mathfrak{X}_{1}$ and $\mathfrak{X}_{2}$ respectively. If $x=x_{1}+x_{2}$ with $x_{i} \in \mathfrak{X}_{i}$ for $i=1$, 2, we define, for every Borel set $e$ of complex numbers, the operator

$$
E(e) x=E_{1}(e) x_{1}+E_{2}(e) x_{2} .
$$

It is clear that $E$ is a countably additive spectral measure and that all the projections $E(e)$ commute with $T$. If $\lambda$ is a complex number not in the closure of the set $e$, then $\lambda I-T$ maps each of the spaces $E_{1}(e) \mathfrak{X}_{1}$ and $E_{2}(e) \mathfrak{X}_{2}$ in a one-to-one manner onto all of itself. Hence $\lambda I-T$ maps $E(e) \mathfrak{X}$ to a one-to-one manner onto all of itself. This shows that $\sigma(T \mid E(e) \mathfrak{X}) \subseteq \bar{e}$ and proves that $E$ is a spectral resolution for $T$ and that $T$ is a spectral operator. Q.E.D.

In most of the remainder of the present section it will be assumed that the spectrum $\sigma(T)$ of $T$ is contained in a closed Jordan curve $\Gamma_{0}$. In order to avoid technical complications it will be convenient to assume also that $\Gamma_{0}$ is smoothly imbedded in a one-parameter family of closed rectifiable Jordan curves. More specifically, and as a basis for the analytical discussion that follows, it will be assumed that there is a function $\xi=\xi(t, \delta)$ which is twice continuously differentiable on its domain $-1 \leqq t, \delta<1$ of definition and which has the following properties. The equation $\xi(-1, \delta)=\xi(+1, \delta)$ holds for all $\delta$ in the interval $-1 \leqq \delta \leqq 1$, whereas $\xi(s, \delta) \neq \xi(t, \delta)$ unless $s=t$ or the pair $s, t$ is the pair $-1,1$. Thus $\xi(\cdot, \delta)$ is the parametric representation of a simple closed rectifiable Jordan curve $\Gamma_{\delta}$. It is assumed that the curves $\Gamma_{\delta}$ are mutually disjoint, that $\Gamma_{\delta_{1}}$ lies inside $\Gamma_{\delta_{2}}$ if $-1 \leqq \delta_{1}<\delta_{2}$ $\leqq 1$, and that $\Gamma_{0}$ contains the spectrum $\sigma(T)$. There will be occasion to integrate around the curve $\Gamma_{\delta}$ with respect to its arc length, and for this reason it is supposed that the curves $\Gamma_{\delta}$ are oriented in the positive sense customary in the theory of complex variables. The simple Jordan arc $\Delta_{\lambda_{0}}$ which is parametrized by the function $\xi\left(t_{0}, \cdot\right)$ is called the transversal through the point $\lambda_{0}=\xi\left(t_{0}, 0\right)$. The principal assumption that will be made throughout most of this section is that the resolvent $R(\lambda ; T)$ has a finite rate of growth as $\lambda$ approaches a 
spectral point $\lambda_{0}$ along the transversal $\Delta_{\lambda_{0}}$ through $\lambda_{0}$. This rate of growth hypothesis is stated formally as follows.

(G) The spectrum of $T$ is contained in the rectifiable Jordan curve $\Gamma_{0}$ described above. Moreover, for each spectral point $\lambda_{0}$ there are two positive integers $\nu=\nu\left(\lambda_{0}\right)$ and $M=M\left(\lambda_{0}\right)$ depending upon $\lambda_{0}$ and such that

$$
\left|\left(\lambda-\lambda_{0}\right)^{\nu} R(\lambda ; T)\right| \leqq M, \quad \lambda \neq \lambda_{0}, \lambda \in \Delta \lambda_{0} .
$$

Although the rate of growth condition (G) will be assumed in most of what follows, it will be stated either explicitly or parenthetically (as was done with the conditions (A), . ., (D)) in any theorem where it is used. It will be seen that this growth condition implies the conditions (A) and (C) and that the boundedness and growth conditions $(B)$ and $(G)$ together come very near to insuring that the operator $T$ is a spectral operator.

Lemma 4. An operator with property (G) also has properties (A) and $(\mathrm{C})$.

Proof. If the operator $T$ in the $B$-space $\mathfrak{X}$ satisfies the growth condition $(G)$, its spectrum lies in the rectifiable Jordan curve $\Gamma_{0}$. Thus the spectrum is nowhere dense and condition (A) follows from Lemma 1.

To prove (C), let $\delta$ be a closed subset of the complex plane and let

$$
\mathfrak{M}(\delta)=\{x \mid x \in \mathfrak{X}, \sigma(x) \subseteq \delta\} .
$$

It will be shown that $M(\delta)$ is closed. For every $x$ we have $\sigma(x)$ $\subseteq \sigma(T) \subseteq \Gamma_{0}$ and thus $M(\delta)=M\left(\delta \Gamma_{0}\right)$ which allows us to assume, with no loss of generality, that $\delta$ is a subset of the curve $\Gamma_{0}$. The set $\delta$ is therefore an intersection $\delta=\bigcap_{\alpha} \delta_{\alpha}$ of sets $\delta_{\alpha}$ each one of which is the complement in $\Gamma_{0}$ of an open subinterval of $\Gamma_{0}$. Since

$$
M(\delta)=M\left(\bigcap_{\alpha} \delta_{\alpha}\right)=\bigcap_{\alpha} M\left(\delta_{\alpha}\right),
$$

in order to see that $M(\delta)$ is closed it will suffice to prove that $M\left(\delta_{\alpha}\right)$ is closed. In other words, we may and shall assume that $\delta$ is the complement of an open subinterval $\gamma$ of $\Gamma_{0}$. Let $\left\{x_{n}\right\}$ be a sequence in $\mathfrak{X}$, convergent to the point $x$, and with $\rho\left(x_{n}\right) \supseteq \gamma$. To prove (C) it will be shown that $\rho(x) \supseteq \gamma$. To do this it is evidently sufficient to show that $\rho(x)$ contains an arbitrary open subinterval $\gamma_{0}$ of $\gamma$. Let $a$ and $b$ be the end points of $\gamma_{0}$ and let $C$ be a simple Jordan curve composed of the transversals $\Delta_{a}, \Delta_{b}$ and arcs connecting their end points in such a way that $C$ includes $\gamma_{0}$ in its interior, intersects $\Gamma_{0}$ only at the points 
$a, b$, and includes the rest of $\Gamma_{0}$ in its exterior.

The condition (G) shows that there is an integer $N$ such that

$$
\lim _{\lambda \rightarrow a ; \lambda \in c}(\lambda-a)^{N}(\lambda-b)^{N} x_{n}(\lambda)=\lim _{\lambda \rightarrow b ; \lambda \in c}(\lambda-a)^{N}(\lambda-b)^{N} x_{n}(\lambda)=0
$$

uniformly in $n=1,2, \cdots$. Thus there are open subarcs $N_{a}, N_{b}$ of $C$ containing $a, b$ respectively and such that the vector $y_{n}(\lambda)$ $=(\lambda-a)^{N}(\lambda-b)^{N} x_{n}(\lambda)$ has norm

$$
\left|y_{n}(\lambda)\right|<\epsilon / 2, \quad n \geqq 1, \quad \lambda \in N_{a} \cup N_{b} .
$$

Since $x_{n} \rightarrow x$ we have

$$
\lim _{n \rightarrow \infty} y_{n}(\lambda)=(\lambda-a)^{N}(\lambda-b)^{N} R(\lambda ; T) x
$$

uniformly for $\lambda$ in $C-N_{a}-N_{b}$. This fact together with the preceding inequality shows that for some integer $n_{0}$ depending upon $\epsilon$ we have

$$
\left|y_{n}(\lambda)-y_{m}(\lambda)\right|<\epsilon, \quad \lambda \in C, n, m \geqq n_{0},
$$

and thus proves that the sequence $\left\{y_{n}(\lambda)\right\}$ converges uniformly for $\lambda$ in $C$. By the maximum modulus principle this sequence converges uniformly on the union of $C$ and its interior to an analytic function $y(\lambda)$. Since

$$
y(\lambda)=\lim _{n} y_{n}(\lambda)=(\lambda-a)^{N}(\lambda-b)^{N} x(\lambda)
$$

for $\lambda \notin \Gamma_{0}$ the vector

$$
X(\lambda)=\frac{y(\lambda)}{(\lambda-a)^{N}(\lambda-b)^{N}}
$$

is an analytic continuation of $x(\lambda)$ into the interior of $C$. Since $(\lambda I-T) x(\lambda)=x$ for $\lambda \notin \Gamma_{0}$, it follows that $(\lambda I-T) X(\lambda)=x$ for all $\lambda$ interior to $C$. Thus $\rho(x)$ includes the interior of $C$ and, since the interior of $C$ includes $\gamma_{0}$, the proof is complete. Q.E.D.

According to the preceding lemma and Theorem 5 of $\$ 3(\mathrm{C})$, an operator $T$ in a weakly complete space will be spectral if it satisfies (B), (G) and (D). We shall now study the condition (D) more carefully and see that the conditions (B) and (G) come very near to implying the condition (D). This will allow us to replace the condition (D), and in a variety of ways, by more satisfactory conditions. For example, it will be seen that an operator $T$ in a reflexive space which satisfies $(G)$ and whose adjoint satisfies (B) is a spectral operator.

Before starting this study it will be convenient to restate the con- 
dition $(\mathrm{G})$ in a form more suitable to the analysis that follows. In the first place, it is clear that the integers $\nu=\nu\left(\lambda_{0}\right)$ and $M=M\left(\lambda_{0}\right)$ with the required properties exist for every $\lambda_{0}$ in $\Gamma_{0}$ even if $\lambda_{0}$ is not in the spectrum. Also, it may be assumed that for each $\lambda_{0}$ in $\Gamma_{0}$ the transversal $\Delta_{\lambda_{0}}$ lies within the circle of radius $1 / 2$ and center $\lambda_{0}$. This shortening of the transversal $\Delta_{\lambda_{0}}$ may be achieved by replacing $\xi(\lambda, \delta)$ by $\xi_{1}\left(\lambda, \delta_{1}\right)$ where $\delta_{1}=K \delta$ with $K$ sufficiently large. Now, if every point of $\Delta_{\lambda_{0}}$ is within a distance of $1 / 2$ from $\lambda_{0}$, it follows from (G) that

$$
\lim _{N \rightarrow \infty}\left(\lambda-\lambda_{0}\right)^{N} R(\lambda ; T)=0
$$

uniformly for $\lambda$ in $\Delta_{\lambda_{0}}$. Thus there is an integer valued function $\nu=\nu\left(\lambda_{0}\right)$ defined for every $\lambda_{0}$ in $\Gamma_{0}$ and such that $\left|\left(\lambda-\lambda_{0}\right)^{\nu\left(\lambda_{0}\right)} R(\lambda ; T)\right|$ $\leqq 1$ for every $\lambda$ in $\Delta_{\lambda_{0}}$ except $\lambda=\lambda_{0}$. In other words the function $M$ $=M\left(\lambda_{0}\right)$ of condition $(\mathrm{G})$ may, without loss of generality, be assumed to be identically one. Thus, condition $(G)$ may be restated in the following equivalent form.

(G) The spectrum of $T$ is contained in the rectifiable Jordan curve $\Gamma_{0}$. Moreover, there is an integer valued function $\nu$ defined on $\Gamma_{0}$ such that for every $\lambda_{0}$ in $\Gamma_{0}$,

$$
\left|\left(\lambda-\lambda_{0}\right) \nu^{\left(\lambda_{0}\right)} R(\lambda ; T)\right| \leqq 1, \quad \lambda \neq \lambda_{0}, \lambda \in \Delta_{\lambda_{0}} .
$$

In the analysis to follow, it is this inequality that will be used rather than the one in the earlier formulation of the growth condition (G).

Definition 5. An integer valued function $\nu$ defined on $\Gamma_{0}$ and satisfying the preceding inequality is called an index function for $T$. An interval of constancy relative to $T$ is a nonvoid open subinterval of $\Gamma_{0}$ upon which some index function for $T$ is constant. A point $\lambda$ in $\Gamma_{0}$ is said to be regular relative to $T$ if it belongs to an interval of constancy and if, in addition, there is an integer $n$ such that the manifold

$$
(\lambda I-T)^{n} \mathfrak{X}+\left\{x \mid(\lambda I-T)^{n} x=0\right\}
$$

is dense in $\mathfrak{X}$.

It should be noted that if

$$
\mathfrak{X}=\mathrm{Cl}(T-\lambda I)^{n} \mathfrak{X}+\left\{x \mid(T-\lambda I)^{n} x=0\right\},
$$

then, by applying the operator $(T-\lambda I)^{n}$ to both sides of this equation, one obtains the inclusion relation

$$
(T-\lambda I)^{n} \mathfrak{X} \subseteq \mathrm{Cl}(T-\lambda I)^{2 n} \mathfrak{X} .
$$


Thus, since the manifolds $(T-\lambda I)^{m \mathfrak{X}}$ decrease as $m$ increases, it follows that

$$
(T-\lambda I)^{n} \mathfrak{X} \subseteq \mathrm{Cl}(T-\lambda I)^{n+1} \mathfrak{X} .
$$

Since the manifolds $\left\{x \mid(T-\lambda I)^{m} x=0\right\}$ increase with $m$, it follows that

$$
(T-\lambda I)^{n+1} \mathfrak{X}+\left\{x \mid(T-\lambda I)^{n+1} x=0\right\}
$$

is dense in $\mathfrak{X}$. By induction it is seen that the manifold

$$
(T-\lambda I)^{n+k \mathfrak{X}}+\left\{x \mid(T-\lambda I)^{n+k} x=0\right\}
$$

is dense in $\mathfrak{X}$ for all $k \geqq 0$. This fact will be stated in the following lemma for future reference.

Lemma 6. A complex number $\lambda$ is regular relative to $T$ if and only if it is contained in an interval of constancy relative to $T$ and, for all suffciently large integers $n$, the manifold

$$
(T-\lambda I)^{n} \mathfrak{X}+\left\{x \mid(T-\lambda I)^{n} x=0\right\}
$$

is dense in $\mathfrak{X}$.

It should also be noted that every point $\lambda$ of $\Gamma_{0}$ which is in the resolvent set of $T$ is regular relative to $T$. This follows since the resolvent set is open and $R(\lambda ; T)$ is continuous so that $\lambda$ is interior to an interval where some index function is constant. Also for $\lambda$ in the resolvent set the density requirement of Definition 5 is satisfied, since for such $\lambda,(T-\lambda I) \mathfrak{X}=\mathfrak{X}$.

Lemma 7. (A) If $\left(\lambda_{0} I-T\right)^{n} x=0$ for some integer $n$ and some $x \neq 0$ then $\sigma(x)=\left\{\lambda_{0}\right\}$.

Proof. Since it is finite, the series

$$
\begin{aligned}
X(\lambda) & =\sum_{j=0}^{\infty} \frac{(-1)^{j}}{\left(\lambda-\lambda_{0}\right)^{j+1}}\left(\lambda_{0} I-T\right)^{j} x \\
& =\sum_{j=0}^{n} \frac{(-1)^{j}}{\left(\lambda-\lambda_{0}\right)^{j+1}}\left(\lambda_{0} I-T\right)^{j} x
\end{aligned}
$$

converges for every $\lambda \neq \lambda_{0}$ and satisfies the equation $(\lambda I-T) X(\lambda)=x$. Thus $X(\lambda)$ is an analytic extension of $R(\lambda ; T) x$ to the complement of $\left\{\lambda_{0}\right\}$. This means that $\sigma(x) \subseteq\left\{\lambda_{0}\right\}$. Since, by Lemma 2 of $\S 3(\mathrm{~A})$, $\sigma(x)$ is not void, we have $\sigma(x)=\left\{\lambda_{0}\right\}$. Q.E.D.

Lemma 8. (B, G) Every closed subinterval of $\Gamma_{0}$ whose end points are egular relative to $T$ belongs to $S_{1}(T)$. 
Proof. Let $\gamma$ be a closed subinterval of $\Gamma_{0}$ whose end points $\lambda_{1}, \lambda_{2}$ are regular relative to $T$. It is clear that, by making a suitable change in the parameter $s$ in the function $\xi(s, \delta)$, it may be assumed that $\lambda_{1}=\xi(-(1 / 2), 0)$ and $\lambda_{2}=\xi(1 / 2,0)$. Since $\lambda_{1}$ and $\lambda_{2}$ are interior to intervals of constancy relative to $T$, there is an $\epsilon>0$ such that for $\left|\lambda_{0}-\lambda_{1}\right|<\epsilon$ or $\left|\lambda_{0}-\lambda_{2}\right|<\epsilon$ the inequality

$$
\left|\lambda-\lambda_{0}\right|^{N}|R(\lambda ; T)| \leqq 1, \quad \lambda_{0} \neq \lambda \in \Delta_{\lambda_{0}}
$$

holds for all sufficiently large values of $N$. In view of Lemma 6 the integer $N$ may be fixed so that the inequality (i) holds and also so that the manifolds

$$
\mathfrak{M}_{i}=\left(\lambda_{i} I-T\right)^{N} \mathfrak{X}+\left\{x \mid\left(\lambda_{i} I-T\right)^{N} x=0\right\}, \quad i=1,2,
$$

are both dense in $\mathfrak{X}$. Since $\mathfrak{M}_{2}$ is dense in $\mathfrak{X}$ the manifold

$$
\left(\lambda_{1} I-T\right)^{N M_{2}}+\left\{x \mid\left(\lambda_{1} I-T\right)^{N} x=0\right\}
$$

is dense in $\mathfrak{X}$, so that

$\left(\lambda_{1} I-T\right)^{N}\left(\lambda_{2} I-T\right)^{N} \mathfrak{X}+\left\{x \mid\left(\lambda_{1} I-T\right)^{N} x=0\right\}+x \mid\left(\lambda_{2} I-T\right)^{N} x=0$

is also dense in $\mathfrak{X}$. By Lemma $7, \sigma(x) \subset \gamma$ if $\left(\lambda_{i} I-T\right)^{N} x=0$ for $i=1$ or $i=2$, so that to prove the present lemma it will be sufficient to show that every element of the form $y=\left(\lambda_{1} I-T\right)^{N}\left(\lambda_{2} I-T\right)^{N} x$ may be approximated arbitrarily closely by a sum $z_{1}+z_{2}$ where $\sigma\left(z_{1}\right)$ is contained in $\gamma$ and $\sigma\left(z_{2}\right)$ is contained in the complement $\gamma^{\prime}$.

Actually more will be proved, for it will be shown that $z_{1}$ and $z_{2}$ may be chosen so that $z_{1}+z_{2}$ is arbitrarily close to $y$ and the spectra $\sigma\left(z_{1}\right), \sigma\left(z_{2}\right)$ are contained in the interior of $\gamma, \gamma^{\prime}$ respectively. Using the operational calculus for analytic functions ${ }^{10}$ we have

$$
\begin{aligned}
y & =\left(\lambda_{1} I-T\right)^{N}\left(\lambda_{2} I-T\right)^{N} x \\
& =\left\{\int_{\Gamma_{1}}-\int_{\Gamma_{-1}}\right\}\left(\lambda_{1}-\lambda\right)^{N}\left(\lambda_{2}-\lambda\right)^{N} R(\lambda ; T) x d \lambda .
\end{aligned}
$$

The transversals $\Delta_{\lambda_{1}}$ and $\Delta_{\lambda_{2}}$ divide the annular region between $\Gamma_{1}$ and $\Gamma_{-1}$ into two simply connected areas, each bounded by a curve consisting of the $\operatorname{arcs} \Delta_{\lambda_{1}}, \Delta_{\lambda_{2}}$ and portions of $\Gamma_{1}, \Gamma_{-1}$. Let the area containing $\gamma$ be called $A_{1}$, and its positively oriented bounding curve, $C_{1}$. Let the area containing the complement $\gamma^{\prime}$ be called $A_{2}$ and its positively oriented bounding curve $C_{2}$. Then, since the integrand in the expression (ii) for $y$ is bounded on $C_{1}$ and $C_{2}$, we have

$$
y=y_{c_{1}}+y_{c_{2}}
$$


where

$$
y_{C_{1}}=\frac{1}{2 \pi i} \int_{C_{i}}\left(\lambda_{1}-\lambda\right)^{N}\left(\lambda_{2}-\lambda\right)^{N} R(\lambda ; T) x d \lambda, \quad i=1,2 .
$$

It will be shown that $y_{C_{1}}$ can be approximated arbitrarily closely by vectors whose spectra lie interior to $C_{1}$, and that $y_{C_{2}}$ can be approximated arbitrarily closely by elements whose spectra lie interior to $C_{2}$. The details will be given only for $y_{C_{2}}$, but the proof for $y_{C_{1}}$ is quite similar.

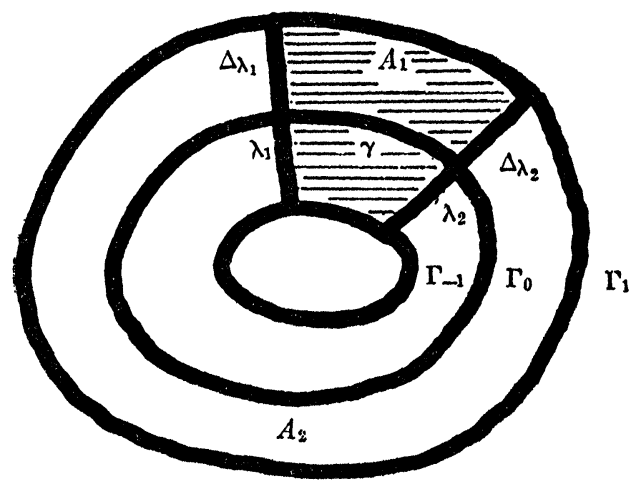

Let $\Delta_{e}^{-}, \Delta_{e}^{+}$be the transversals through the points $\xi((-1 / 2)-\epsilon, 0)$, $\xi((1 / 2)+\epsilon, 0)$ respectively. Let $A_{1}^{\epsilon}, A_{2}^{\epsilon}$ be the two simply connected regions into which the annular region between $\Gamma_{1}$ and $\Gamma_{-1}$ is divided by the transversals $\Delta_{e}^{-}$and $\Delta_{\epsilon}^{+}$. Let $A_{2}^{\epsilon}$ be that one of these areas contained in $A_{2}$, and let $C_{2}^{e}$ be its bounding curve. In view of (i) the integrand in (iii) is uniformly bounded, and it follows from an elementary calculation using Lebesgue's dominated convergence theorem that

$$
y_{C_{2}}=\lim _{\epsilon \rightarrow 0} y_{C_{2}^{e}}
$$

where

$$
y_{C_{2}}=\frac{1}{2 \pi i} \int_{C_{2}}\left(\lambda_{1}-\lambda\right)^{N}\left(\lambda_{2}-\lambda\right)^{N} R(\lambda ; T) x d \lambda .
$$

Thus, to see that $y_{C_{2}}$ is the limit of a sequence of vectors whose spectra are interior to $C_{2}$, it will suffice to show the spectrum $\sigma\left(y_{C_{2}^{\epsilon}}\right)$ is contained in $A_{2}^{\epsilon}$. It is evident that the integral 


$$
I(\xi)=\frac{1}{2 \pi i} \int_{C}{ }_{C}\left(\lambda_{1}-\lambda\right)^{N}\left(\lambda_{2}-\lambda\right)^{N}(\xi-\lambda)^{-1} R(\lambda ; T) x d \lambda
$$

is analytic for $\xi$ outside $A_{2}^{\bullet}$. Since

$$
\begin{aligned}
(\xi I-T) I(\xi) & =\frac{1}{2 \pi i} \int_{C_{2}^{\epsilon}}\left(\lambda_{1}-\lambda\right)^{N}\left(\lambda_{2}-\lambda\right)^{N}(\xi-\lambda)^{-1}(\xi I-T) R(\lambda ; T) x d \lambda \\
& =\frac{1}{2 \pi i} \int_{C_{2}^{\epsilon}}\left(\lambda_{1}-\lambda\right)^{N}\left(\lambda_{2}-\lambda\right)^{N}(\xi-\lambda)^{-1}(\xi-\lambda) R(\lambda ; T) x d \lambda \\
& =y_{C_{2}}^{\epsilon},
\end{aligned}
$$

it follows that $I(\xi)$ is an analytic extension of $R(\xi ; T) y_{C_{2}}$. Thus $\rho\left(y_{C_{2}}^{e}\right)$ includes the entire complement of $A_{2}^{e}$ and so the spectrum $\sigma\left(y_{c_{2}^{\epsilon}}^{\epsilon}\right)$ is contained in $A_{2}^{\bullet}$. Q.E.D.

Corollary 9. $(\mathrm{B}, \mathrm{G})$ Let $\gamma$ be a closed subinterval of $\Gamma_{0}$ whose end points $\lambda_{1}, \lambda_{2}$ belong to intervals of constancy relative to $T$. Then, for sufficiently large $N$, the manifold $\left(\lambda_{1} I-T\right)^{N}\left(\lambda_{2} I-T\right)^{N} \mathfrak{X}$ is contained in the closed manifold determined by vectors of the form $z_{1}+z_{2}$ where $\sigma\left(z_{1}\right)$ is interior to $\gamma$ and $\sigma\left(z_{2}\right)$ is interior to the complementary arc $\gamma^{\prime}$.

PRoof. The above statement is what was actually proved in the preceding proof. Q.E.D.

LEMma 10. (B, G) If the set of points regular relative to $T$ is dense on $\Gamma_{0}$ then every closed subinterval of $\Gamma_{0}$ whose end points are regular relative to $T$ is in $S(T)$ and every Borel subset of the plane is measurable $T$.

PROof. Let $\gamma$ be a closed subinterval of $\Gamma_{0}$ whose end points are regular relative to $T$. Since the points regular relative to $T$ are dense in $\Gamma_{0}$, there is an increasing sequence $\left\{\gamma_{n}\right\}$ of open subintervals of $\Gamma_{0}$ each member of which has regular end points in the arc $\gamma^{\prime}$ complementary to $\gamma$ and whose union is the whole arc $\gamma^{\prime}$. By Lemma 8 the intervals $\gamma$ and $\bar{\gamma}_{n}, n=1,2, \cdots$, are all in $S_{1}(T)$. Since $\gamma$ is in $S_{1}(T)$ there are, for each $x$ in $\mathfrak{X}$ and $\epsilon>0$, vectors $y$ and $z$ such that

$$
|y+z-x|<\epsilon, \quad \sigma(y) \subseteq \gamma, \sigma(z) \subseteq \gamma^{\prime} .
$$

Since $\sigma(z)$ is compact we have $\bar{\gamma}_{n} \supseteq \gamma_{n} \supseteq \sigma(z)$ for all sufficiently large $n$. Thus, by Lemma 2 of $\S 3(\mathrm{~B}),\left[E(\gamma)+E\left(\bar{\gamma}_{n}\right)\right](y+z)=y+z$ for all large $n$. By the boundedness assumption (B) the norms of the projections $E(\gamma)+E\left(\bar{\gamma}_{n}\right)$ are bounded in $n$ and, since $\epsilon>0$ is arbitrary, we have

$$
x=\lim _{n}\left[E(\gamma) x+E\left(\bar{\gamma}_{n}\right)\right] x,
$$
$x \in X$. 
Now it follows from Lemma 4 that $T$ has the properties (A) and (C), and thus it is seen from Lemma 2 of $\$ 3(\mathrm{C})$ that

$$
\sigma(E(\gamma) x) \subseteq \gamma \sigma(x), \quad \sigma\left(E\left(\bar{\gamma}_{n}\right) x\right) \subseteq \bar{\gamma}_{n} \sigma(x) .
$$

These relations (i) and (ii) show that $\gamma$ is in $S_{2}(T)$. Since $\gamma$ is an arbitrary closed subinterval of $\Gamma_{0}$ whose end points are regular relative to $T$, this proves that the closure $\bar{\gamma}_{n}$ is also in $S_{2}(T)$. Thus it follows from (i) that $\gamma$ is in $S(T)$. It follows that every Borel subset of $\Gamma_{0}$ is measurable $T$. By Lemma 9 of $\S 3(\mathrm{~B})$ every subset of $\rho(T)$ is in $S(T)$ and hence is a set measurable $T$. Thus, since $\sigma(T) \subseteq \Gamma_{0}$, every Borel set in the plane is measurable T. Q.E.D.

In view of Lemma 10 it behooves us to seek conditions under which points on $\Gamma_{0}$ which are regular relative to $T$ are dense on $\Gamma_{0}$. Some results in this direction will be found in the next four lemmas.

LEMMA 11. (G) The union of all intervals of constancy relative to $T$ is an open set dense in $\Gamma_{0}$.

Proof. It is clear that the union of intervals of constancy is open. To see that it is dense let $\gamma$ be a closed subarc of $\Gamma_{0}$ having positive length and let

$$
\begin{aligned}
\gamma_{n} & =\left\{\lambda_{0}\left|\lambda_{0} \in \gamma,\right| \lambda-\left.\lambda_{0}\right|^{n}|R(\lambda ; T)| \leqq 1, \lambda_{0} \neq \lambda \in \Delta_{\lambda_{0}}\right\} \\
& =\left\{\lambda_{0}\left|\lambda_{0} \in \gamma,\right| \xi\left(\lambda_{0}, \delta\right)-\left.\lambda_{0}\right|^{n} R\left(\xi\left(\lambda_{0}, \delta\right) ; T\right)|\leqq 1,0<| \delta \mid \leqq 1\right\} .
\end{aligned}
$$

It is clear from the second expression for $\gamma_{n}$ that it is closed, and it follows from (G) that every point in $\gamma$ is in one of the sets $\gamma_{n}$. Thus, by the Baire category theorem one of the sets $\gamma_{n}$ contains a nontrivial subinterval of $\gamma$.Q.E.D.

LEmma 12. (G) If the point spectrum of the adjoint $T^{*}$ contains no nontrivial subarc of $\Gamma_{0}$ then the set of points regular relative to $T$ is dense in $\Gamma_{0}$.

Proof. If $\lambda$ is not in the point spectrum of the adjoint of $T$ then there is no functional $x^{*} \neq 0$ for which $x^{*}(\lambda I-T) \mathfrak{X}=0$. In view of the Hahn-Banach theorem this means that $(\lambda I-T) \mathfrak{X}$ is dense in $\mathfrak{X}$. Hence if $\lambda$ is also in an interval of constancy for $T$ then $\lambda$ is regular relative to $T$. The present lemma thus follows from Lemma 11. Q.E.D.

Lemma 13. (G) If the space $\mathfrak{X}$ is reflexive and if the function identically one on $\Gamma_{0}$ is an index function for $T$ then every point of $\Gamma_{0}$ is regular relative to $T$.

PROOF. It is clear that $\Gamma_{0}$ itself is an interval of constancy relative 
to $T$. Let $x$ be an arbitrary vector in $\mathfrak{X}, \lambda_{0}$ an arbitrary point on $\Gamma_{0}$ and let $\left\{\lambda_{n}\right\}$ be a sequence of points in the transversal $\Delta_{\lambda_{0}}$ with $\lambda_{n} \neq \lambda_{0}$ and $\lambda_{0}=\lim \lambda_{n}$. Since $\mathfrak{X}$ is reflexive, the bounded

$$
\left\{\left(\lambda_{n}-\lambda_{0}\right) R\left(\lambda_{n} ; T\right) x\right\}
$$

contains a subsequence weakly convergent to an element $y$ of $\mathfrak{X}$. By replacing the sequence $\left\{\lambda_{n}\right\}$ by a suitably chosen subsequence it may therefore be assumed that, in the weak operator topology, we have

$$
\lim _{n}\left(\lambda_{n}-\lambda_{0}\right) R\left(\lambda_{n} ; T\right) x=y .
$$

Then $\left(\lambda_{0} I-T\right) y$ is the weak limit of

$$
\left(\lambda_{n}-\lambda_{0}\right)\left(\lambda_{0} I-T\right) R\left(\lambda_{n} ; T\right) x=\left(\lambda_{n}-\lambda_{0}\right) x-\left(\lambda_{0}-\lambda_{n}\right)^{2} R\left(\lambda_{n} ; T\right) x
$$

and this limit is clearly zero. Thus $\left(\lambda_{0} I-T\right) y=0$.

It will next be shown that the vector $x-y$ is in the closure of the manifold $\left(\lambda_{0} I-T\right) \mathfrak{X}$. To see this it will, in view of the Hahn-Banach theorem, suffice to show that $x^{*}(x-y)=0$ for every linear functional $x^{*}$ which vanishes on $\left(\lambda_{0} I-T\right) \mathfrak{X}$. If $x^{*}$ is such a functional then $T^{*} x^{*}=\lambda_{0} x^{*}$,

$$
R\left(\lambda_{n}: T\right)^{*} x^{*}=\frac{x^{*}}{\lambda_{n}-\lambda_{0}}
$$

and so

$$
x^{*} y=\lim _{n \rightarrow \infty} x^{*}\left(\lambda_{n}-\lambda_{0}\right) R\left(\lambda_{n} ; T\right) x=x^{*} x,
$$

and $x^{*}(x-y)=0$. It has been shown that an arbitrary vector $x$ in $\mathfrak{X}$ is the sum of a vector $y$ with $\left(\lambda_{0} I-T\right) y=0$ and a vector $x-y$ in the closure of $\left(\lambda_{0} I-T\right) \mathfrak{X}$. Since $\lambda_{0}$ is interior to an interval of constancy relative to $T$ it is therefore a regular point relative to $T$. Q.E.D.

Lemma 14 . (G) If $\mathfrak{X}$ is reflexive and if the adjoint $T^{*}$ satisfies the boundedness condition (B) then the regular points relative to $T$ are dense in $\Gamma_{0}$ and, in particular, every interval of constancy relative to $T$ consists entirely of regular points.

Proof. In view of Lemma 11 it suffices to show that a point $\lambda_{0}$ in an interval of constancy relative to $T$ is regular. Since $\sigma\left(T^{*}\right)=\sigma(T)$ and $R\left(\lambda ; T^{*}\right)=R(\lambda ; T)^{*}$, it follows that $T^{*}$ also satisfies the growth condition $(G)$ and that every index function for $T$ is also an index function for $T^{*}$ and vice versa. By applying Corollary 9 to $T^{*}$ and 
the interval consisting of the single point $\lambda_{0}$ it is seen that, for $N$ sufficiently large, every element in the manifold $\left(\lambda_{0} I^{*}-T^{*}\right)^{N} \mathfrak{X}^{*}$ may be approximated by elements $z^{*}$ with $\lambda_{0} \notin \sigma\left(z^{*}\right)$. To prove that $\lambda_{0}$ is regular relative to $T$ it will be shown that, for such $N$, the manifold

$$
\left.\left(\lambda_{0} I-T\right)^{N} \mathfrak{X}+\left\{x \mid \lambda_{0} I-T\right)^{N} x=0\right\}
$$

is dense in $\mathfrak{X}$. To do this it will, in view of the Hahn-Banach theorem, suffice to show that the functional $x^{*}=0$ is the only functional which vanishes on the manifold (i). Let $x^{*}$ be such a functional. Then

$$
\left(\lambda_{0} I^{*}-T^{*}\right)^{N} x^{*}=0 .
$$

To see that $x^{*}=0$ it will first be shown that

$$
x^{*} \in \mathrm{Cl}\left(\lambda_{0} I^{*}-T^{*}\right)^{N \mathfrak{X}^{*}} \text {. }
$$

If (iii) is not true then, since $\mathfrak{X}$ is reflexive, it follows from the HahnBanach theorem that there is an $x$ in $\mathfrak{X}$ with $x^{*} x \neq 0$ and $\left[\left(\lambda_{0} I^{*}\right.\right.$ $\left.\left.-T^{*}\right)^{N} \mathfrak{X}^{*}\right] x=0$ which means that $\left(\lambda_{0} I-T\right)^{N} x=0$. Since $x^{*}$ vanishes on the manifold (i) we have $x^{*} x=0$ which contradicts the inequality $x^{*} x \neq 0$ and establishes (iii).

Now from (ii) and (iii) together it may be concluded that $x^{*}=0$. To do this note first that, according to Lemma $7, \sigma\left(x^{*}\right)=\left\{\lambda_{0}\right\}$. By Corollary 9, applied to $T^{*}$ and the interval consisting of the single point $\lambda_{0}$, there is a sequence $\left\{x_{n}^{*}\right\}$ converging to $x^{*}$ with $\lambda_{0} \notin \sigma\left(x_{n}^{*}\right)$. Since $T^{*}$ satisfies the condition (B) we have

$$
\left|x^{*}\right| \leqq K\left|x^{*}-x_{n}^{*}\right| \rightarrow 0,
$$

and so $x^{*}=0$. This completes the proof that the manifold (i) is dense in $\mathfrak{X}$ and thus proves that $\lambda_{0}$ is regular relative to T. Q.E.D.

The preceding lemmas, when combined with the general criteria given in Theorems 5 and 7 of $\S 3(\mathrm{C})$, allow us to summarize a set of conditions that are sufficient to guarantee that an operator is a spectral operator. This will be done in the following two results.

THEOREM 15. If a bounded linear operator in a weakly complete space satisfies the boundedness condition (B) and the growth condition (G) then it is a spectral operator provided that any one of the following conditions holds.

(a) The point spectrum of the adjoint contains no nontrivial subarc of $\Gamma_{0}$.

(b) The space is reflexive and the function

$$
\nu(\lambda)=1,
$$$$
\lambda \in \Gamma_{0}
$$ 
is an index function for the operator.

(c) The space is reflexive and the adjoint operator satisfies the boundedness condition (B).

Proof. If the bounded linear operator $T$ in a weakly complete space has the properties (B) and (G) then, by Lemma 4 , it has the properties (A) and (C). Thus, in view of Theorem 5 of $\$ 3(C)$, to prove the present theorem it suffices to show that $T$ has property (D). According to Lemma 10 the condition (D) will be satisfied if the points regular relative to $T$ are dense on $\Gamma_{0}$. Thus Lemmas $12,13,14$ give the desired conclusions. Q.E.D.

THEOREM 16. Let $T$ be a bounded linear operator in the B-space $\mathfrak{X}$ which satisfies the conditions (B) and (G) and let $B$ be the field of Borel sets in the plane. Then the adjoint $T^{*}$ is a spectral operator of class $(B, \mathfrak{X})$ provided that any one of the conditions (a), (b), (c) of the preceding theorem hold.

Proof. The proof is the same as that of the preceding theorem except that Theorem 7 of $\S 3(\mathrm{C})$ is used instead of Theorem 5 .

It is to be expected from analogies with finite matrices that a spectral operator $T$ which satisfies the growth condition $(G)$ will be a spectral operator of type $m-1$ if and only if the constant function $\nu(\lambda) \equiv m$ is an index function. For convenience of reference this finite rate of growth condition is stated formally as follows.

Definition 17. The bounded linear operator $T$ is said to satisfy the growth condition $\left(G_{m}\right)$ if its spectrum lies in the curve $\Gamma_{0}$ and if for some constant $M$,

$$
\left|\left(\xi-\xi_{\delta}\right)^{m} R\left(\xi_{\delta} ; T\right)\right| \leqq M, \quad \xi \in \Gamma_{0}, 0<|\delta| \leqq 1,
$$

where $\xi_{\delta}$ is the intersection of the transversal $\Delta_{\xi}$ with the curve $\Gamma_{\delta}$.

Theorem 18. A bounded linear operator $T$ in Hilbert space whose spectrum lies in the Jordan curve $\Gamma_{0}$ will be a spectral opertor of type $m-1$ if and only if both $T$ and its adjoint satisfy the conditions (B) and $\left(\mathrm{G}_{m}\right)$.

Proof. If $T$ is a spectral operator in the Hilbert space $\mathfrak{X}$ then, since $\mathfrak{X}$ is reflexive, the adjoint $T^{*}$ is, by Lemma 6 of $\S 3(\mathrm{C})$, a spectral operator. By Theorem 4 of $\S 3(\mathrm{C})$, both $T$ and $T^{*}$ satisfy the boundedness condition (B). Now if $T$ is of type $m-1$ with radical part $N$ and resolution of the identity $E$ then

$$
R(\xi ; T)=\sum_{n=0}^{m-1} N^{n} \int_{\sigma(T)} \frac{E(d \lambda)}{(\xi-\lambda)^{n+1}}, \quad \xi \in \rho(T),
$$


from which it is apparent that $T$ and consequently $T^{*}$ also satisfy the condition $\left(\mathrm{G}_{m}\right)$.

Conversely, let $T$ and its adjoint satisfy the conditions (B) and $\left(\mathrm{G}_{m}\right)$. It is clear from Theorem $15(\mathrm{c})$ that $T$ is a spectral operator and so it suffices to prove only that $T$ is of type $m-1$. The proof of this will require the following lemma.

Lemma 19. Let $T$ be a spectral operator in Hilbert space $\mathfrak{S}$ and let $E$ be its resolution of the identity. Then there is a constant $K$ such that for any finite collection $A_{j}, j=1,2, \cdots, n$, of bounded operators in $\mathfrak{W}$ which commute with $T$, and any collection $\sigma_{j}, j=1,2, \cdots, n$, of disjoint Borel sets, we have

$$
\left|\sum_{j=1}^{n} A_{j} E\left(\sigma_{j}\right)\right| \leqq K \sup _{1 \leq j \leq n}\left|A_{j}\right| .
$$

Proof. According to the Lorch-Mackey-Wermer result there is a linear one-to-one map $B$ with $B \mathfrak{S}=\mathfrak{S}$, with $B$ and $B^{-1}$ both continuous and such that for each Borel set $\sigma$ the projection

$$
P(\sigma)=B E(\sigma) B^{-1}
$$

is self-adjoint. If $B_{j}=B A_{j} B^{-1}$ then

$$
B\left\{\sum_{j=1}^{n} A_{j} E\left(\sigma_{j}\right)\right\} B^{-1}=\sum_{j=1}^{n} B_{j} P\left(\sigma_{j}\right) .
$$

Since $A_{j}$ commutes with $T$ it commutes with $E(\sigma)$ and hence $B_{j}$ commutes with $P(\sigma)$. Thus

$$
\begin{aligned}
\left|\sum_{j=1}^{n} B_{j} P\left(\sigma_{j}\right) x\right|^{2} & =\left|\sum_{j=1}^{n} P\left(\sigma_{j}\right) B_{j} x\right|^{2}=\sum_{j=1}^{n}\left|P\left(\sigma_{j}\right) B_{j} x\right|^{2} \\
& \leqq \sup _{j}\left|B_{j}\right|^{2} \sum_{j=1}^{n}\left|P\left(\sigma_{j}\right) x\right|^{2} \\
& \leqq \sup _{j}\left|B_{j}\right|^{2}|x|^{2}
\end{aligned}
$$

which proves the lemma. Q.E.D.

The remaining part of the proof of Theorem 18 will involve Riemann integrals of the type

$$
\int_{\sigma(T)} F(\xi) E(d \xi)
$$

where $E$ is the resolution of the identity for $T$ and $F$ is an operator valued function defined on $\sigma(T)$, continuous in the uniform operator 
topology, and for which

$$
F(\xi) T=T F(\xi),
$$

$\xi \in \sigma(T)$.

It follows that $F(\xi)$ also commutes with the projections in the range of $E$, i.e.,

$$
F(\xi) E(\sigma)=E(\sigma) F(\xi), \quad \xi \in \sigma(T),
$$

for every Borel set $\sigma$. If $\pi=\left(\sigma_{1}, \cdots, \sigma_{n}\right), \pi^{\prime}=\left(\sigma_{1}^{\prime}, \cdots, \sigma_{n}^{\prime}\right)$ are two partitions of $\sigma(T)$ and if $\xi_{1} \in \sigma_{i}, \xi_{j}^{\prime} \in \sigma_{j}^{\prime}, i=1, \cdots, n, j=1, \cdots, n^{\prime}$, then using (iii) and Lemma 19 , it is seen that, for some constant $K$,

$$
\begin{aligned}
& \mid \sum_{i=1}^{n} F\left(\xi_{i}\right) E\left(\sigma_{i}\right)-\sum_{j=1}^{n^{\prime}} F\left(\xi_{j}^{\prime}\right) E\left(\sigma_{j}^{\prime}\right) \\
& =\mid \sum_{i=1}^{n} \sum_{j=1}^{n^{\prime}}\left\{F\left(\xi_{i}\right)-F_{j}\left(\xi_{j}^{\prime}\right)\right\} E\left(\sigma_{i} \sigma_{j}^{\prime}\right) \\
& \quad \leqq K \sup \left|F\left(\xi_{i}\right)-F\left(\xi_{j}^{\prime}\right)\right|,
\end{aligned}
$$

where the supremum is taken over those $i$ and $j$ for which $\sigma_{i} \sigma_{j}^{\prime}$ is not void. If by the norm $|\pi|$ is understood the quantity

$$
|\pi|=\max _{1 \leq i \leq n} \operatorname{diam} \sigma_{i}
$$

it is seen that the limit

$$
\lim _{|\pi| \rightarrow 0} \sum_{i=1}^{n} F\left(\xi_{i}\right) E\left(\sigma_{i}\right)
$$

exists in the uniform topology of operators for every function $F$ on $\sigma(T)$ which is continuous in the uniform operator topology and satisfies (ii). This limit defines the Riemann integral (i). It is clear that the integral is linear in $F$ and satisfies the inequality

$$
\left|\int_{\sigma(T)} F(\xi) E(d \xi)\right| \leqq K \sup _{\xi \in \sigma(T)}|F(\xi)| \text {. }
$$

If $F$ and $G$ are two continuous operator valued functions both satisfying (ii) then, since

$$
\left(\sum_{i=1}^{n} F\left(\xi_{i}\right) E\left(\sigma_{i}\right)\right)\left(\sum_{j=1}^{n} G\left(\xi_{j}\right) E\left(\sigma_{j}\right)\right)=\sum_{i=1}^{n} F\left(\xi_{i}\right) G\left(\xi_{i}\right) E\left(\sigma_{i}\right),
$$

it follows that

(v) $\left\{\int_{\sigma(T)} F(\xi) E(d \xi)\right\}\left\{\int_{\sigma(T)} G(\xi) E(d \xi)\right\}=\int_{\sigma(T)} F(\xi) G(\xi) E(d \xi)$. 


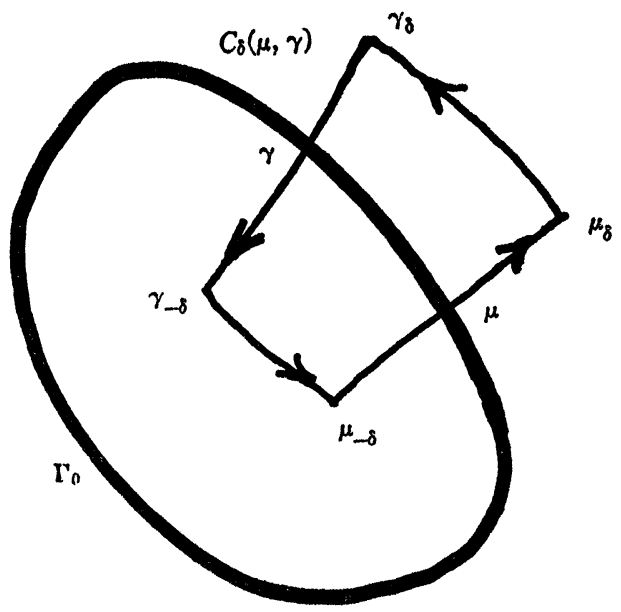

It will next be shown that

$$
\int_{\sigma(T)}(\xi I-T)^{2 m} E(d \xi)=0
$$

The proof of (vi) will use the integral

$$
I(\mu, \gamma)=\frac{1}{2 \pi i} \int_{C_{\delta}(\mu, \gamma)}(\mu-\xi)^{m}(\gamma-\xi)^{m} R(\xi ; T) d \xi
$$

where $\mu, \gamma$ are points on $\Gamma_{0}$ and, for $0<\delta \leqq 1$, the contour $C_{\delta}(\mu, \gamma)$ is the positively oriented contour through $\mu, \gamma$ which is defined as follows. For an arbitrary $\xi$ in $\Gamma_{0}$ let $\xi_{\delta}$ be the intersection of the transversal through $\xi$ with the curve $\Gamma_{\delta}$. The curve $C_{\delta}(\mu, \gamma)$ is a positively oriented Jordan curve through the points $\mu_{\delta}, \gamma_{\delta}, \gamma_{-\delta}, \mu_{-\delta}$ and consisting of subarcs of the curves $\Gamma_{\delta}, \Delta_{\gamma}, \Gamma_{-\delta}, \Delta_{\mu}$. The curve $C_{\delta}(\mu, \gamma)$ is defined so as to contain in its interior the interior of the directed segment $(\mu, \gamma)$ on the oriented contour $\Gamma_{0}$. The integrand $f(\xi)$ in $I(\mu, \gamma)$ is defined and continuous at every point of $C_{\delta}(\mu, \gamma)$ except at $\mu$ and $\gamma$. In view of the condition $\left(\mathrm{G}_{m}\right), f(\xi)$ is bounded on $C_{\delta}(\mu, \gamma)$. Hence $I(\mu, \gamma)$ exists and is clearly independent of $\delta$ since $f(\xi)$ has its only singularities on $\Gamma_{0}$. It will first be shown that $I(\mu, \gamma) \rightarrow 0$ as $|\mu-\gamma| \rightarrow 0$. Let $\epsilon>0$ be arbitrary and fix $\delta>0$ so that the $\operatorname{arcs} \mu_{-\delta} \mu_{\delta}$ and $\gamma_{-\delta} \gamma_{\delta}$ both have length less than $\epsilon$. Fix $K_{\epsilon}$ so that

$$
|R(\xi ; T)|=K_{\epsilon}, \quad \xi \in \Gamma_{\delta} \cup \Sigma_{-\delta}
$$


and fix $\alpha_{\epsilon}>0$ so that the arcs $\gamma_{-\delta} \mu_{-\delta}$ and $\mu_{\delta} \gamma_{\delta}$ both have length less than $\epsilon / K_{\epsilon}$ provided that $|\mu-\gamma|<\alpha_{\epsilon}$. Then, for $|\mu-\gamma|<\alpha_{\epsilon}$, we have

$$
|I(\mu, \gamma)| \leqq \frac{1}{2 \pi}\left[2 \epsilon M+2 K_{\epsilon} \epsilon / K_{\epsilon}\right]
$$

which shows that $I(\mu, \gamma) \rightarrow 0$ as $|\mu-\gamma| \rightarrow 0$.

It will next be observed that the spectrum of any vector of the form $I(\mu, \gamma) x$ is contained in the directed closed subarc $\mu \gamma$. To see this let $\lambda$ lie in $\rho(T)$ and outside $C_{\delta}(\mu, \gamma)$. Then, it is seen from the resolvent equation that

$$
R(\lambda ; T) I(\mu, \gamma) x=\frac{1}{2 \pi i} \int_{C_{\delta}(\mu, \gamma)} \frac{(\mu-\xi)^{m}(\gamma-\xi)^{m}}{\lambda-\xi} R(\xi ; T) x d \xi
$$

and the integral on the right of this equation gives an analytic extension of $R(\lambda ; T) I(\mu, \gamma) x$ to all points outside of $C_{\delta}(\mu, \gamma)$. Thus the spectrum $\sigma(I(\mu, \gamma) x)$ is contained in the closed arc $\mu \gamma$. Thus if the closed intervals $\mu \gamma$ and $\mu^{\prime} \gamma^{\prime}$ of $\Gamma_{0}$ are disjoint we have from Lemma 2 of $\S 3(\mathrm{~A})$,

$$
E(\mu \gamma) I\left(\mu^{\prime}, \gamma^{\prime}\right)=0 .
$$

Let $\mu_{n}, \mu, \gamma, \gamma_{n}$ be an ordered set on the oriented curve $\Gamma_{0}$ with $\mu_{n} \rightarrow \mu$ and $\gamma_{n} \rightarrow \gamma$. Then $E(\mu \gamma) I\left(\gamma_{n}, \mu_{n}\right)=0$. Since

$$
(\mu I-T)^{m}(\gamma I-T)^{m}=I\left(\mu_{n}, \mu\right)+I(\mu, \gamma)+I\left(\gamma, \gamma_{n}\right)+I\left(\gamma_{n}, \mu_{n}\right),
$$

and $I\left(\mu_{n}, \mu\right) \rightarrow 0, I\left(\gamma, \gamma_{n}\right) \rightarrow 0$, it follows that

$$
E(\mu \gamma)(\mu I-T)^{m}(\gamma I-T)^{m}=I(\mu, \gamma) .
$$

Now let $\Gamma_{0}$ be divided into $n$ nonoverlapping subintervals $\sigma_{1}, \cdots, \sigma_{n}$ each of length $L / n$ where $L$ is the length of $\Gamma_{0}$, and let $\xi_{1}, \xi_{2}, \cdots, \xi_{n}$, $\xi_{n+1}=\xi_{1}$ be their positively ordered sequence of end points. Then, according to the preceding equation

$$
E\left(\sigma_{j}\right)\left(\xi_{j} I-T\right)^{m}\left(\xi_{j+1} I-T\right)^{m}=I\left(\xi_{j}, \xi_{j+1}\right) .
$$

Now let $\delta=1 / n$ so that the length of $C_{\delta}\left(\xi_{j}, \xi_{j+1}\right)$ is of the order of $1 / n$. It follows from (vii) and (viii) that

$$
E\left(\sigma_{j}\right)\left(\xi_{j} I-T\right)^{m}\left(\xi_{j+1} I-T\right)^{m}=\frac{C_{1}}{n^{m+1}},
$$


and thus that

$$
\left|\sum_{j=1}^{n}\left(\xi_{j} I-T\right)^{m}\left(\xi_{j+1} I-T\right)^{m} E\left(\sigma_{j}\right)\right|=\frac{C_{2}}{n^{m}} .
$$

An elementary continuity argument shows that the sum whose norm appears on the left of the preceding inequality approaches the integral $\int_{\sigma(T)}(\xi I-T)^{2 m} E(d \xi)$ as $n \rightarrow \infty$ and thus, this inequality establishes the Equation (vi).

It will next be proved that

$$
\int_{\sigma(T)}(\xi I-T)^{i} E(d \xi)=0 \quad j \geqq m .
$$

In view of (vi) this equation may be proved by induction downward. Thus it will be shown that (ix) holds for the integer $j \geqq m$ provided that it holds for the integer $j+1$. To do this let $\xi_{\delta}$ be the point of intersection of the curve $\Gamma_{\delta}$ with the transversal $\Delta_{\xi}$ through the point $\xi$ on $\Gamma_{0}$ and let $R\left(\xi_{\delta}\right)=R\left(\xi_{\delta} ; T\right)$. Then, from (iv) and the hypothesis $\left(G_{m}\right)$ we have

$$
\left|\int_{\sigma(T)}\left(\xi-\xi_{\delta}\right)^{j+1} R\left(\xi_{\delta}\right) E(d \xi)=C_{1}\right| \xi-\xi_{\delta} \mid
$$

which shows that

$$
\lim _{\delta \rightarrow 0} \int_{\sigma(T)}\left(\xi-\xi_{\delta}\right)^{j+1} R\left(\xi_{\delta}\right) E(d \xi)=0 .
$$

On the other hand it is seen by writing $\left(\xi-\xi_{\delta}\right) I=(\xi I-T)-\left(\xi_{\delta} I-T\right)$ that

$$
\begin{aligned}
\int_{\sigma(T)}\left(\xi-\xi_{\delta}\right)^{j+1} R\left(\xi_{\delta}\right) E(d \xi) & =\int_{\sigma(T)}\left(\xi_{\delta} I-T\right)^{j+1} R\left(\xi_{\delta}\right) E(d \xi) \\
& +\sum_{r=1}^{j+1}(-1)^{r}\left(\begin{array}{c}
j+1 \\
r
\end{array}\right) \int_{\sigma(T)}(\xi I-T)^{j+1-r}\left(\xi_{\delta} I-T\right)^{r-1} E(d \xi) .
\end{aligned}
$$

But, in view of (v) and the induction hypothesis,

$$
\int_{\sigma\left(T^{\prime}\right)}(\xi I-T)^{j+1} R\left(\xi_{\delta}\right) E(d \xi)=0, \quad 0<\delta \leqq 1
$$


Thus, since $\lim _{\delta \rightarrow 0}\left(\xi_{\delta} I-T\right)^{r-1}=(\xi I-T)^{r-1}$ in the uniform operator topology it follows from (iv) that

$$
\lim _{\delta \rightarrow 0} \int_{\sigma(T)}\left(\xi-\xi_{\delta}\right)^{j+1} R\left(\xi_{\delta}\right) E(d \xi)=-\int_{\sigma(T)}(\xi I-T)^{j} E(d \xi)
$$

which establishes the equation (ix) for every $j \geqq m$. Now if $S$ is the scalar part of $T$ then

$$
\begin{aligned}
0 & =\int_{\sigma(T)}(\xi I-T)^{m} E(d \xi)=\sum_{r=0}^{m}\left(\begin{array}{c}
m \\
r
\end{array}\right) \int_{\sigma(T)} \xi^{m-r} E(d \xi)(-T)^{r} \\
& =\sum_{r=0}^{m}\left(\begin{array}{c}
m \\
r
\end{array}\right) S^{m-r}(-T)^{r}=(S-T)^{m}=(-N)^{m},
\end{aligned}
$$

which shows that $N^{m}=0$. The fact that $T$ is of type $m-1$ is now evident. Q.E.D.

The spectral theorem for a bounded selfadjoint operator $T$ in Hilbert space follows from Theorem 18. A well known and elementary argument shows that the spectrum is real and that the resolvent has first order rate of growth along vertical lines. This shows that the condition $\left(\mathrm{G}_{m}\right)$ is satisfied with $m=1$. Now, since $|x+y|^{2}=|x|^{2}$ $+|y|^{2}$ for orthogonal vectors, to verify the condition (B) it will suffice to show that $x$ and $y$ are orthogonal if their spectra are disjoint. In this case the function

$$
(R(\lambda ; T) x, y)=(x, R(\bar{\lambda} ; T) y)=\operatorname{conj}(R(\bar{\lambda} ; T) y, x)
$$

the complex conjugate of $(R(\bar{\lambda} ; T) y, x)$, is analytic for $\lambda \notin \sigma(x)$ and $\bar{\lambda} \notin \sigma(y)$. Since $\sigma(y)$ is real and disjoint with $\sigma(x)$ the function

$$
(R(\lambda: T) x, y)=\frac{(x, y)}{\lambda}+\frac{(T x, y)}{\lambda^{2}}+\cdots
$$

is everywhere analytic and vanishes at infinity. Hence it is identically zero and the coefficient $(x, y)=0$. This verification of the condition (B) is due to Schwartz. Another one may be found in [12].

Another special case is useful in the discussion of the nonselfadjoint second order singular differential boundary value problems discussed by Neumark. Because of its special nature however, an independent proof, shorter than that of Theorem 18 is possible and will be given here. In this theorem the operator $T$ has its spectrum in the smooth curve $\Gamma_{0}$ as described in the discussion of the growth condition $(\mathrm{G})$. It will not be necessary however to assume the growth condition as it will follow from the other assumptions. 
THEOREM 20. Let the operator $T$ in the reflexive space $\mathfrak{X}$ have its spectrum in the curve $\Gamma_{0}$. In addition let $\mathfrak{X}_{0}, \mathfrak{X}_{0}^{*}$ be dense linear manifolds in the spaces $\mathfrak{X}, \mathfrak{X}^{*}$ respectively with the following three properties.

(i) For $x_{0}$ in $\mathfrak{X}_{0}$ and $x_{0}^{*}$ in $\mathfrak{X}_{0}^{*}$ there is a constant $K\left(x_{0}^{*}, x_{0}\right)$ with

$$
\left|x_{0}^{*} R(\xi(t, \delta) ; T) x_{0}\right| \leqq K\left(x_{0}^{*}, x_{0}\right), \quad-1 \leqq t, \delta \leqq 1, \delta \neq 0 .
$$

(ii) For each $x_{0}$ in $\mathfrak{X}_{0}$ and $x_{0}^{*}$ in $\mathfrak{X}_{0}^{*}$ the limits

$$
\begin{aligned}
& R^{+}\left(\lambda, x_{0}^{*}, x_{0}\right)=\lim _{\delta \rightarrow 0^{+}} x_{0}^{*} R(\xi(t, \delta) ; T) x_{0}, \\
& R^{-}\left(\lambda, x_{0}^{*}, x_{0}\right)=\lim _{\delta \rightarrow 0^{-}} x_{0}^{*} R(\xi(t, \delta) ; T) x_{0},
\end{aligned}
$$

exist for each point $\lambda=\xi(t, 0)$ in $\Gamma_{0}$.

(iii) There is a constant $M$ depending only upon $T$ such that

$$
\int_{\sigma(T)}\left|R^{+}\left(\lambda, x_{0}^{*}, x_{0}\right)-R^{-}\left(\lambda, x_{0}^{*}, x_{0}\right)\right| d s \leqq M\left|x_{0}^{*}\right|\left|x_{0}\right|,
$$

$x_{0} \in \mathfrak{X}, x_{0}^{*} \in \mathfrak{X}^{*}$,

where $s$ is the arc length on $\Gamma_{0}$.

Then $T$ is a scalar type spectral operator whose spectral resolution is given by the formula

$$
x_{0}^{*} E(\sigma) x_{0}=\frac{1}{2 \pi i} \int_{\sigma}\left\{R^{+}\left(\lambda, x_{0}^{*}, x_{0}\right)-R^{-}\left(\lambda, x_{0}^{*}, x_{0}\right)\right\} d \lambda .
$$

Proof. Let $\delta>0$ and let $f$ be single valued and analytic in a neighborhood of the annular region bounded by the curves $\Gamma_{\delta}$ and $\Gamma_{-\delta}$. Then ${ }^{10}$

$$
f(T)=\frac{1}{2 \pi i}\left[\int_{\Gamma_{\delta}} f(\lambda) R(\lambda ; T) d \lambda-\int_{\Gamma_{-\delta}} f(\lambda) R(\lambda ; T) d \lambda\right],
$$

where both integrals are taken in the positive sense. Then, in view of the hypotheses (i) and (ii), this formula may be written as

$$
\begin{array}{r}
x_{0}^{*} f(T) x_{0}=\frac{1}{2 \pi i} \int_{\Gamma_{0}} f(\lambda)\left\{R^{+}\left(\lambda, x_{0}^{*}, x_{0}\right)-R^{-}\left(\lambda, x_{0}^{*}, x_{0}\right)\right\} d \lambda, \\
x_{0} \in \mathfrak{X}_{0}, x_{0}^{*} \in \mathfrak{X}_{0}^{*},
\end{array}
$$

or, since the integrand vanishes if $\lambda$ is in the resolvent set,

(v) $x_{0}^{*} f(T) x_{0}=\frac{1}{2 \pi i} \int_{\sigma(T)} f(\lambda)\left\{R^{+}\left(\lambda, x_{0}^{*}, x_{0}\right)-R^{-}\left(\lambda, x_{0}^{*}, x_{0}\right)\right\} d \lambda$,

$$
x_{0} \in \mathfrak{X}_{0}, x_{0}^{*} \in \mathfrak{X}_{0}^{*}
$$


In view of the hypothesis (iii) the right side of this equation (v) defines, for each bounded Borel function $f$ on $\sigma(T)$, a continuous bilinear form $\left(f, x_{0}^{*}, x_{0}\right)$ which, since $\mathfrak{X}_{0}$ and $\mathfrak{X}_{0}^{*}$ are dense, has a unique extension to a bounded bilinear form $\left(f, x^{*}, x\right)$ defined for all $x$ in $\mathfrak{X}$ and $x^{*}$ in $\mathfrak{X}^{*}$. Since $\mathfrak{X}$ is reflexive it follows that there is a unique operator $f(T)$ in $\mathfrak{X}$ for which (v) holds. The mapping $f \rightarrow f(T)$ is a homomorphism on the algebra of analytic functions $f$ and since every continuous function on $\Gamma_{0}$ is the uniform limit of analytic functions it follows that this map is also a homomorphism on the algebra of continuous functions. To see that it is a homomorphism on the algebra of bounded Borel functions note that for a fixed continuous function $g$ the set of all bounded Borel functions $f$ for which

$$
(f g)(T)=f(T) g(T),
$$

includes all continuous functions. Furthermore, if the equation (vi) holds for each function in a uniformly bounded pointwise convergent sequence $\left\{f_{n}\right\}$ then it follows from (v) that it holds for the limit function $f=\lim f_{n}$. This shows that (vi) holds for every bounded Borel function $f$ and every continuous function $g$. A repetition of this argument shows that it also holds if $f$ and $g$ are both bounded Borel functions. Thus the operators $f(T)$ and $g(T)$ commute and also, as (v) shows, satisfy the inequality

$$
|f(T)| \leqq \sup _{\lambda \in \sigma(T)}|f(\lambda)|
$$

These facts show that the operator $E(\sigma)$ defined by (iv) is a bounded spectral measure which, in view of the Orlicz-Pettis theorem, is countably additive in the strong operator topology. To see that $E$ is a spectral resolution for $T$ it will therefore be sufficient to show that, for each Borel subset $\sigma$ of $\sigma(T)$, the spectrum of the restriction $T \mid E(\sigma) \mathfrak{X}$ is contained in $\bar{\sigma}$. For every $\xi \notin \bar{\sigma}$ let the bounded Borel function $r_{\xi}$ be defined by the equation $r_{\xi}(\lambda)=(\xi-\lambda)^{-1} \chi_{\sigma}(\lambda)$ where $\chi_{\sigma}$ is the characteristic function of $\sigma$. Then $r_{\xi}(T)(\xi I-T)=E(\sigma)$ which shows that $(T \mid E(\sigma) \mathfrak{X}) \subseteq \bar{\sigma}$, and proves that $T$ is a spectral operator. It follows from (vii) that $T$ is a scalar operator. Q.E.D.

\section{REFERENCES}

1. W. G. Bade, Unbounded spectral operators, Pacific J. Math. vol. 4 (1954) pp. 373-392.

2. - Weak and strong limits of spectral operators, Pacific J. Math vol. 4 (1954) pp. 393-413.

3. - On Boolean algebras of projections and algebras of operators, Trans. Amer. Math. Soc. vol. 80 (1955) pp. 345-360. 
4. - A multiplicity theory for Boolean algebras of projections in Banach spaces, O.N.R. Technical Report No. 18, University of California, Berkeley, Calif., 1958.

5. G. D. Birkhoff, Boundary value and expansion problems of ordinary linear differential equations, Trans. Amer. Math. Soc. vol. 9 (1908) pp. 373-395.

6. J. Dieudonné, Sur la theorie spectrale, J. Math. Pures Appl. (9) vol. 35 (1956) pp. 175-187. 651.

7. N. Dunford, Spectral theory, Bull. Amer. Math. Soc. vol. 49 (1943) pp. 637-

8. - Spectral theory I. Convergence to projections, Trans. Amer. Math. Soc. vol. 54 (1943) pp. 185-217.

9. - Spectral theory in abstract spaces and Banach algebras, Proc. Symposium on Spectral Theory and Differential Problems, 1951, pp. 1-65.

10. - Spectral theory, ibid., 1951, pp. 203-208.

11. - The reduction problem in spectral theory, Proceedings of the International Congress of Mathematicians, Cambridge, Mass., 1950, vol. 2 (1952) pp. 115122.

12. - Spectral theory II. Resolutions of the identity, Pacific J. Math. vol. 2 (1952) pp. 559-614.

13. - Spectral operators, Pacific J. Math. vol. 4 (1954) pp. 321-354.

14. N. Dunford and J. T. Schwartz, Linear operators, Part I, General theory, Interscience Publishers, New York, 1958.

15. - Linear operators, Part II. Spectral theory (to be published by Interscience Publishers.)

16. S. R. Foguel, Sums and products of commuting spectral operators, Ark. Mat. (1957) pp. 449-461.

17. - The relations between a spectral operator and its scalar part (to appear in the Pacific J. Math.).

18. - Normal operators of finite multiplicity (to appear in Comm. Pure Appl. Math.).

19. - A perturbation theorem for spectral operators (to appear in Comm. Pure Appl. Math.).

20. I. Fredholm, Sur une classe d'equations fonctionnelles, Acta Math. vol. 27 (1903) pp. 365-390.

21. K. O. Friedrichs, On the perturbation of continuous spectra, Comm. Pure Appl. Math. vol. 1 (1948) pp. 361-406.

22. T. H. Hildebrandt, Über vollstetige lineare Transformationen, Acta Math. vol. 51 (1928) pp. 311-318.

23. S. Kakutani, An example concerning uniform boundedness of spectral measures, Pacific J. Math. vol. 4 (1954) pp. 363-372.

24. H. P. Kramer, Perturbation of differential operators, Pacific J. Math. vol. 7 (1957) pp. 1405-1434.

25. E. R. Lorch, Bicontinuous linear transformations in certain vector spaces, Bull. Amer. Math. Soc. vol. 45 (1939) pp. 564-569.

26. G. W. Mackey, Commutative Banach algebras, Mimeographed lecture notes, Harvard University, 1952.

27. J. Moser, Störungstheorie des kontinuierlichen Spektrums für gewöhnliche Differentialgleichungen zweiter Ordnung, Math. Ann. vol. 125 (1953) pp. 366-393.

28. M. A. Neumark, Investigation of the spectrum and expansion in eigenfunctions of singular nonselfadjoint differential operators of the second order, Uspehi Mat. 
Nauk (N.S.) vol. 8 no. 4 (56) (1953) pp. 174-175 (Russian), Math. Rev. vol. 15 (1954) p. 530.

29. - On expansion in characteristic functions of non-self-adjoint singular differential operators of the second order, Dokl. Akad. Nauk SSSR vol. 89 (1953) pp. 213-216 (Russian), Math. Rev. vol. 15 (1954) p. 33.

30. - Investigation of the spectrum and the expansion in eigenfunctions of a non-self-adjoint operator of the second order on a series-axis, Trudy Moskov. Mat. Obšc. vol. 3 (1954) pp. 181-270 (Russian), Math. Rev. vol. 15 (1954) p. 959.

31. B. J. Pettis, On integration in vector spaces, Trans. Amer. Math. Soc. vol. 44 (1938) pp. 277-304.

32. F. Riesz, Über lineare Funktionalgleichungen, Acta Math. vol. 41 (1918) pp. 71-98.

33. J. Schauder, Über lineare, vollstetige Funktional-operationen, Studia Math. vol. 2 (1930) pp. 183-196.

34. J. T. Schwartz, Perturbations of spectral operators, and applications. I, Pacific J. Math. vol. 4 (1954) pp. 415-458.

35. - Two perturbation formulae, Comm. Pure Appl. Math. vol. 8 (1955) pp. 371-376.

36. D. R. Smart, Eigenfunction expansions in $L^{p}$ and $C$ (to appear in Illinois J. Math.).

37. J. Wermer, Commuting spectral measures on Hilbert space, Pacific J. Math. vol. 4 (1954) pp. 355-361.

38. — On restrictions of operators, Proc. Amer. Math. Soc. vol. 4 (1953) pp. 860-865.

YALE UNIVERSITY 